\title{
Ray Scattering by an Arbitrarily Oriented Spheroid .II. Transmission and Cross-Polarization Effects
}

James A. Lock

Cleveland State University, j.lock@csuohio.edu

Follow this and additional works at: https://engagedscholarship.csuohio.edu/sciphysics_facpub

Part of the Physics Commons

How does access to this work benefit you? Let us know!

\section{Publisher's Statement}

This paper was published in Applied Optics and is made available as an electronic reprint with the permission of OSA. The paper can be found at the following URL on the OSA website: http://www.opticsinfobase.org/ao/abstract.cfm?URI=ao-35-3-515. Systematic or multiple reproduction or distribution to multiple locations via electronic or other means is prohibited and is subject to penalties under law.

\section{Original Citation}

Lock, James A. "Ray Scattering by an Arbitrarily Oriented Spheroid .II. Transmission and CrossPolarization Effects." Applied Optics 35 (1996): 515-531.

\section{Repository Citation}

Lock, James A., "Ray Scattering by an Arbitrarily Oriented Spheroid .II. Transmission and Cross-Polarization Effects" (1996). Physics Faculty Publications. 42.

https://engagedscholarship.csuohio.edu/sciphysics_facpub/42

This Article is brought to you for free and open access by the Physics Department at EngagedScholarship@CSU. It has been accepted for inclusion in Physics Faculty Publications by an authorized administrator of EngagedScholarship@CSU. For more information, please contact library.es@csuohio.edu. 


\title{
Ray scattering by an arbitrarily oriented spheroid. II. Transmission and cross-polarization effects
}

\author{
James A. Lock
}

\begin{abstract}
Transmission of an arbitrarily polarized plane wave by an arbitrarily oriented spheroid in the short-wavelength limit is considered in the context of ray theory. The transmitted electric field is added to the diffracted plus reflected ray-theory electric field that was previously derived to obtain an approximation to the far-zone scattered intensity in the forward hemisphere. Two different types of cross-polarization effects are found. These are (a) a rotation of the polarization state of the transmitted rays from when they are referenced with respect to their entrance into the spheroid to when they are referenced with respect to their exit from it and $(b)$ a rotation of the polarization state of the transmitted rays when they are referenced with respect to the polarization state of the diffracted plus reflected rays. (1) 1996 Optical Society of America
\end{abstract}

\section{Introduction}

A number of optical particle-sizing instruments, such as the phase-Doppler particle analyzer, measure the diameter of small particles by analyzing certain features of their forward-hemisphere lightscattering signature. ${ }^{1,2}$ For particles much larger than the wavelength of light, forward-hemisphere scattering is well approximated by ray theory, which considers the diffracted, reflected, and transmitted rays that reach the detector. For scattering by a sphere, the ray-theory far-zone scattered intensity closely matches the results of Lorenz-Mie theory ${ }^{3,4}$ for particle-size parameters as low as 30 and for scattering angles as large as $50^{\circ}$. As a result of this close match, phase-Doppler particle analyzer calibration curves have been based almost entirely on ray theory. $1,2,5,6$

A basic assumption underlying the calculation of optical sizing-instrument calibration curves is that the particles being sized are spherical. A necessary first step in evaluating the response of these instruments to nonspherical particles is to have an accurate and easily implemented theory of light scattering by such particles. Because the simplest nonspherical particle is a spheroid, understanding scattering by a spheroid should be helpful in determining the impor-

The author is with the Department of Physics, Cleveland State University, Cleveland, Ohio 44115.

Received 2 February 1995; revised manuscript received 1 June 1995.

0003-6935/96/030515-17\$06.00/0

(1) 1996 Optical Society of America tant features of scattering by nonspherical particles in general.

There are a number of different methods for exactly solving the electromagnetic boundary-value problem of a plane wave scattered by a spheroidal particle. ${ }^{7-11}$ The numerical implementation of these methods, however, suffers from ill conditioning for size parameters greater than approximately 35 and for large spheroid eccentricities. . $^{8,11,12}$ The raytheory model of spheroid scattering is an attractive alternative because it is expected to provide a reasonable approximation to the solution of the exact wave-scattering problem for particle-size parameters beginning approximately where the numerical implementation of the exact methods starts becoming ill conditioned. Ray scattering by a spheroid has already been applied to the analysis of the generalized rainbow caustic in the backward hemisphere, which is caused by the confluence of a number of rays making one internal reflection within the spheroid before exiting. ${ }^{13-19}$ Ray-tracing programs for scattering by an arbitrarily shaped particle have also been developed for certain specialized applications. ${ }^{20,21}$

The purpose of this paper, along with a companion paper $^{22}$ that is hereafter designated as part I, is to describe scattering in the forward hemisphere of an arbitrarily polarized plane wave by an arbitrarily oriented dielectric spheroid by the use of ray theory. In part I diffraction and specular reflection were considered. In this paper transmission, which is the third of the physical processes expected to dominate scattering in the forward hemisphere, is consid- 
ered. Diffraction plus reflection was exactly soluble in the sense that the magnitude, phase, and polarization of the electric field of the outgoing rays were directly expressible in terms of the scattering angles $\Theta$ and $\Phi$. This is not the case for transmission because of the complexity of both the refraction geometry and the spheroid shape. If the plane wave is not incident parallel to the spheroid major axis, another complexity occurs as well. For this case the plane of incidence of a ray at its point of entrance on the lit side of the spheroid does not coincide with the plane of incidence at its point of exit. This leads to cross-polarization effects that do not occur for scattering by a dielectric sphere. ${ }^{23-25}$ For example, a transmitted ray that was incident upon the spheroid with the transverse electric (TE) polarization will exit it elliptically polarized, i.e., with a mixture of TE and transverse magnetic (TM) polarizations. Similarly, the TE polarization directions of the transmitted and reflected rays reaching an observer at the scattering angles $\Theta, \Phi$ are rotated with respect to each other. The transmitted ray is additionally elliptically polarized when referenced with respect to the TE and TM polarization directions of the reflected ray.

Cross-polarization effects also occur for scattering by a nonspherical particle much smaller than the wavelength of light because of differences in the particle's polarizability in different directions. ${ }^{26,27}$ Similar effects also occur for an optically active particle. ${ }^{28}$ Cross-polarization effects originating solely from the geometry of the particle also occur for scattering by a dielectric cylinder at diagonal incidence. ${ }^{29}$ For scattering by a spheroid, the crosspolarization intensity has been computed with the exact solution to the wave-scattering problem..$^{30}$ Our purpose here is to demonstrate clearly and explicitly the geometric origin of the cross-polarization effects in the context of ray theory.

The body of this paper proceeds as follows. Section 2 presents a brief review of both the notation and the spheroid geometry that were described in detail in part I. In Subsections 3.A.-3.C. the magnitude, phase, and polarization vector of the transmitted electric field are obtained. In Section 4 these results, are combined with the diffracted plus reflected electric field obtained in part I, and the cross-polarization effects are examined. Last, in Section 5 the diffracted plus reflected plus transmitted far-zone intensity is computed. Our results are then compared with those obtained by the use of other methods.

\section{Spheroid Geometry}

Consider a plane wave of wavelength $\lambda$, wave number

$$
k=\frac{2 \pi}{\lambda},
$$

and angular frequency $\omega$ propagating along the positive $z$ axis of an $x y z$ coordinate system fixed in the lab reference frame. The electric field of the plane wave is

$$
\mathbf{E}_{\text {inc }}=E_{0}\left(\cos \chi \hat{u}_{x}+\sin \chi \hat{u}_{y}\right) \exp (i k z-i \omega t) .
$$

The plane wave is scattered by an arbitrarily oriented spheroid. The transmitted contribution to the outgoing electric field in the far zone at the scattering angles $\Theta, \Phi$ is of the form

$$
\begin{aligned}
\mathbf{E}_{\text {trans }}(\Theta, \Phi)= & \frac{i E_{0}}{k R} \exp (i k R-i \omega t) S_{\text {trans }}(\Theta, \Phi) \\
& \times \exp \left[i \delta_{\text {trans }}(\Theta, \Phi)\right] \boldsymbol{\epsilon}_{\text {trans }}(\Theta, \Phi) .
\end{aligned}
$$

In Eq. (3) $R$ is the distance from the center of the spheroid at the origin of coordinates to the observer; $S_{\text {trans }}$ is the magnitude of the transmitted electric field, ignoring the Fresnel coefficients at the spheroid surface; $\delta_{\text {trans }}$ is the phase of the transmitted electric field with respect to that of the reference ray of Subsection 3.D. of part I; and $\boldsymbol{\epsilon}_{\text {trans }}$ is the polarization vector of the transmitted electric field and contains the Fresnel coefficients. The dielectric spheroid has the real refractive index $n>1$. There is no other restriction on the numerical value of $n$. The surface of the spheroid is given by

$$
\frac{x^{\prime \prime 2}}{a^{2}}+\frac{y^{\prime \prime 2}}{a^{2}}+\frac{z^{\prime \prime 2}}{b^{2}}=1
$$

where the $x^{\prime \prime} y^{\prime \prime} z^{\prime \prime}$ axes are attached to the spheroid. The spheroid $z^{\prime \prime}$ axis lies in the $\theta, \phi$ direction with respect to the $x y z$ lab coordinate system, where $0 \leq$ $\theta \leq \pi / 2$ and $0 \leq \phi<2 \pi$. The description of scattering by the spheroid is simplified considerably if we use a second lab coordinate system $x^{\prime} y^{\prime} z^{\prime}$ rotated with respect to the $x y z$ lab system by the angle $\phi$ about the $z=z^{\prime}$ axis. In this new coordinate system the equation of the lit (i.e., lower) and the shadowed (i.e., upper) spheroid surface is

$$
\left.\begin{array}{c}
z_{\text {upper }}{ }^{\prime} \\
z_{\text {lower }}^{\prime}
\end{array}\right\}=w A r^{\prime} \cos \xi^{\prime} \pm \frac{a b}{A}\left(1-r^{\prime 2}\right)^{1 / 2},
$$

where

$$
\begin{aligned}
& w=\sin \theta \cos \theta\left(\frac{b^{2}-a^{2}}{A^{2}}\right), \\
& A=\left(b^{2} \sin ^{2} \theta+a^{2} \cos ^{2} \theta\right)^{1 / 2}, \\
& B=a,
\end{aligned}
$$

and where the elliptical coordinate $r^{\prime}$ and $\xi^{\prime}$ are defined by

$$
\begin{aligned}
& x^{\prime}=A r^{\prime} \cos \xi^{\prime}, \\
& y^{\prime}=B r^{\prime} \sin \xi^{\prime},
\end{aligned}
$$


with $0 \leq r^{\prime} \leq 1$ and $0 \leq \xi^{\prime}<2 \pi$. The outward unit normal to a point on the lit surface of the spheroid, $\hat{m}^{\prime}$, and the outward unit normal to a point on the shadowed surface, $\hat{n}^{\prime}$, are shown in figure 4 of part I, and their equations are given by equations 14 and 15 of part I.

\section{A. Magnitude of the Transmitted Electric Field}

The trajectory of a ray transmitted through the arbitrarily oriented spheroid is shown in Fig. 1. All quantities pertaining to the ray as it enters the spheroid on the lit surface have the subscript 0 , all quantities pertaining to the ray inside the spheroid have the subscripts 01 , and all quantities pertaining to the ray's exit from the spheroid have the subscript 1. An incident ray is parameterized by the rotated lab frame coordinates $r_{0}{ }^{\prime}$ and $\xi_{0}{ }^{\prime}$. The unit wave vector of an incident ray is

$$
\hat{k}_{i 0}=\hat{u}_{z}{ }^{\prime} .
$$

The unit normal $\hat{m}_{0}{ }^{\prime}$ to the lit surface at the point of entrance of the ray has the spherical angles $\pi-\Psi_{0}$, $\eta_{0}$ with respect to the $x^{\prime} y^{\prime} z^{\prime}$ axes, i.e.,

$$
\hat{m}_{0}{ }^{\prime}=\sin \Psi_{0} \cos \eta_{0} \hat{u}_{x}{ }^{\prime}+\sin \Psi_{0} \sin \eta_{0} \hat{u}_{y}{ }^{\prime}-\cos \Psi_{0} \hat{u}_{z}{ }^{\prime} .
$$

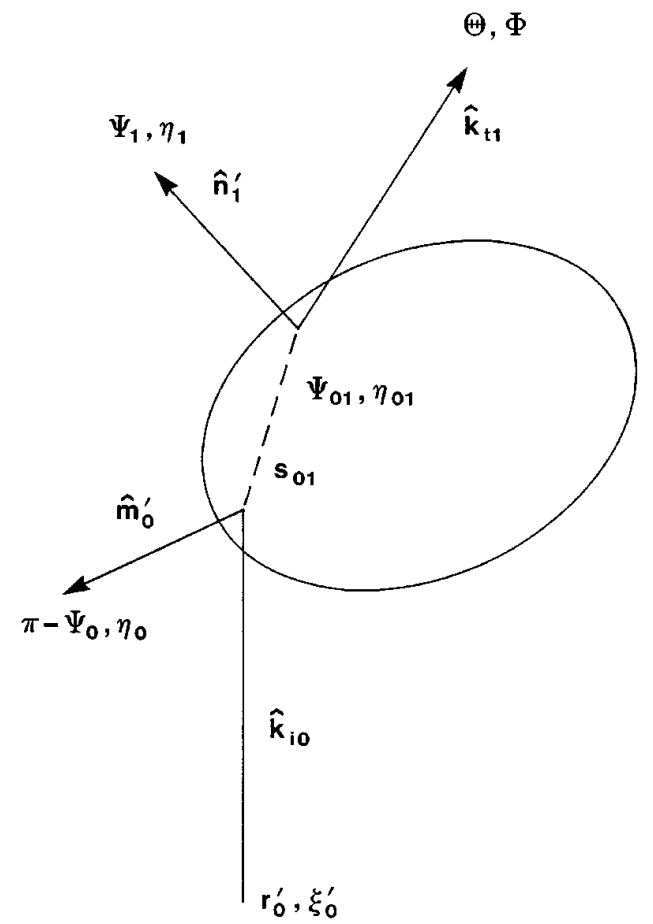

Fig. 1. Geometry of the transmitted ray. The unit normal $\hat{m}_{0}{ }^{\prime}$ to the surface at the point of entrance is in the $\pi-\Psi_{0}, \eta_{0}$ direction with respect to the $x^{\prime} y^{\prime} z^{\prime}$ rotated lab coordinate system. The portion of the ray inside the spheroid has length $s_{01}$ and is in the $\Psi_{01}, \eta_{01}$ direction. The unit normal $\hat{n}_{1}{ }^{\prime}$ to the surface at the point of exit on the shadowed side of the spheroid is in the $\Psi_{1}, \eta_{1}$ direction.
Using Eq. (14) of part I and Eq. (5) above, we obtain

$$
\tan \eta_{0}=\frac{\frac{a b}{A B} q_{0}{ }^{\prime} \sin \xi_{0}{ }^{\prime}}{\frac{a b}{A^{2}} q_{0}{ }^{\prime} \cos \xi_{0}{ }^{\prime}+w},
$$

$$
\tan ^{2}\left(\pi-\Psi_{0}\right)=\left(\frac{a b}{A^{2}} q_{0}{ }^{\prime} \cos \xi_{0}{ }^{\prime}+w\right)^{2}+\left(\frac{a b}{A B} q_{0}{ }^{\prime} \sin \xi_{0}{ }^{\prime}\right)^{2}
$$

with

$$
q_{0}{ }^{\prime}=\frac{r_{0}{ }^{\prime}}{\left(1-r_{0}{ }^{2}\right)^{1 / 2}}
$$

These results are analogous to equations 21,23 , and 24 of part I for the normal to the lit surface at the point of incidence of the reflected ray.

After transmission into the spheroid, the ray propagates in the $\Psi_{01}, \eta_{01}$ direction with respect to the $x^{\prime} y^{\prime} z^{\prime}$ axes. The unit transmitted wave vector is given by

$$
\begin{aligned}
\hat{k}_{t 0}= & \sin \Psi_{01} \cos \eta_{01} \hat{u}_{x}{ }^{\prime}+\sin \Psi_{01} \sin \eta_{01} \hat{u}_{y}{ }^{\prime} \\
& +\cos \Psi_{01} \hat{u}_{z}{ }^{\prime} .
\end{aligned}
$$

It may also be written s $^{31}$

$$
\hat{k}_{t 0}=\frac{1}{n} \hat{k}_{i 0}+\left(\frac{\cos \theta_{i 0}}{n}-\cos \theta_{t 0}\right) \hat{m}_{0}{ }^{\prime},
$$

where

$$
\theta_{i 0}=\Psi_{0}
$$

is the angle of incidence of the ray at the lit surface. The angle of refraction $\theta_{t 0}$ is given by Snell's law

$$
n \sin \theta_{t 0}=\sin \Psi_{0} .
$$

A comparison of Eqs. (15) and (16) allows us to relate the angles of $\hat{k}_{t 0}$ to the angles of $\hat{m}_{0}{ }^{\prime}$. We obtain

$$
\begin{aligned}
& \Psi_{01}=\Psi_{0}-\theta_{t 0} \\
& \eta_{01}=\eta_{0}+\pi .
\end{aligned}
$$

The relation between $\eta_{01}$ and $\eta_{0}$ is expected on physical grounds. The plane of incidence containing $\hat{k}_{i 0}$ and $\hat{m}_{0}{ }^{\prime}$ makes an angle $\eta_{0}$ with respect to the $x^{\prime}$ axis, so $\hat{k}_{t 0}$ must also lie in this plane. Furthermore, Fig. 1 suggests that $\hat{m}_{0}{ }^{\prime}$ and $\hat{k}_{t 0}$ lie on opposite sides of the $z^{\prime}$ axis in the $\eta_{0}$ plane accounting for the difference of $\pi$ between $\eta_{0}$ and $\eta_{01}$.

The trajectory of the ray inside the spheroid is 
given by

$$
\begin{aligned}
& x^{\prime}=x_{0}{ }^{\prime}-s_{01} \sin \Psi_{01} \cos \eta_{0}, \\
& y^{\prime}=y_{0}{ }^{\prime}-s_{01} \sin \Psi_{01} \sin \eta_{0}, \\
& z^{\prime}=z_{0}{ }^{\prime}+s_{01} \cos \Psi_{01},
\end{aligned}
$$

where $s_{01} \geq 0$. The transmitted ray exits the spheroid on either its shadowed or its lit surface. By combining Eqs. (5) and (20) we find that the distance of travel of the ray inside the spheroid for either possibility is ray sometimes occurs on the lit surface. For this case, combining Eqs. (5) and (22) gives

$$
\begin{aligned}
s_{01}\left(\cos \Psi_{01}+w \sin \Psi_{01} \cos \eta_{0}\right) & -\frac{a b}{A}\left(1-r_{0}{ }^{2}\right)^{1 / 2} \\
= & -\frac{a b}{A}\left(1-{r_{1}}^{\prime 2}\right)^{1 / 2} .
\end{aligned}
$$

Thus the sign of the left-hand side of Eqs. (25) and (26) may be used as an indicator as to which side of the spheroid the transmitted ray exists.

$$
s_{01}=\frac{2 a b}{A}\left(1-r_{0}{ }^{\prime}\right)^{1 / 2}\left[\frac{\left(\cos \Psi_{01}+w \sin \Psi_{01} \cos \eta_{0}\right)+\frac{a b}{A B} q_{0}{ }^{\prime} \sin \Psi_{01}\left(\sin \xi_{0}{ }^{\prime} \sin \eta_{0}+\frac{B}{A} \cos \xi_{0}{ }^{\prime} \cos \eta_{0}\right)}{\left(\cos \Psi_{01}+w \sin \Psi_{01} \cos \eta_{0}\right)^{2}+\frac{a^{2} b^{2}}{A^{2} B^{2}} \sin ^{2} \Psi_{01}\left(\sin ^{2} \eta_{0}+\frac{B^{2}}{A^{2}} \cos ^{2} \eta_{0}\right)}\right] .
$$

The coordinates of the ray at its point of exit from the spheroid are found by combining Eqs. (20) and (21) to give

$$
\begin{aligned}
& x_{1}{ }^{\prime}=x_{0}{ }^{\prime}-s_{01} \sin \Psi_{01} \cos \eta_{0}=A r_{1}{ }^{\prime} \cos \xi_{1}{ }^{\prime}, \\
& y_{1}{ }^{\prime}=y_{0}{ }^{\prime}-s_{01} \sin \Psi_{01} \sin \eta_{0}=B r_{1}{ }^{\prime} \sin \xi_{1}{ }^{\prime}, \\
& z_{1}{ }^{\prime}=z_{0}{ }^{\prime}+s_{01} \cos \Psi_{01},
\end{aligned}
$$

which is equivalent to

$$
\begin{aligned}
r_{1}{ }^{2}= & r_{0}{ }^{2}-\frac{2 r_{0}{ }^{\prime}}{B} s_{01} \sin \Psi_{01} \\
& \times\left(\sin \xi_{0}{ }^{\prime} \sin \eta_{0}+\frac{B}{A} \cos \xi_{0}{ }^{\prime} \cos \eta_{0}\right) \\
& +\frac{s_{01}{ }^{2}}{B^{2}} \sin ^{2} \Psi_{01}\left(\sin ^{2} \eta_{0}+\frac{B^{2}}{A^{2}} \cos ^{2} \eta_{0}\right), \\
\tan \xi_{1}{ }^{\prime}= & \frac{r_{0}{ }^{\prime} \sin \xi_{0}{ }^{\prime}-\frac{s_{01}}{B} \sin \Psi_{01} \sin \eta_{0}}{r_{0}{ }^{\prime} \cos \xi_{0}{ }^{\prime}-\frac{s_{01}}{A} \sin \Psi_{01} \cos \eta_{0}}
\end{aligned}
$$

For most commonly encountered values of $b / a$ and $n$, the transmitted ray exits the spheroid on its shadowed side. For this case, combining Eqs. (5) and (22) gives

$$
\begin{aligned}
s_{01}\left(\cos \Psi_{01}+w \sin \Psi_{01} \cos \eta_{0}\right) & -\frac{a b}{A}\left(1-{r_{0}}^{\prime 2}\right)^{1 / 2} \\
& =\frac{a b}{A}\left(1-{r_{1}}^{\prime 2}\right)^{1 / 2} .
\end{aligned}
$$

But for a spheroid with high eccentricity and large refractive index, the point of exit of the transmitted
Let us assume for the moment that the ray exits on the upper or shadowed side. The unit normal $\hat{n}_{1}{ }^{\prime}$ to the spheroid at the point of exit has the spherical angles $\Psi_{1}, \eta_{1}$ with respect to the $x^{\prime} y^{\prime} z^{\prime}$ axes, i.e.,

$\hat{n}_{1}{ }^{\prime}=\sin \Psi_{1} \cos \eta_{1} \hat{u}_{x}{ }^{\prime}+\sin \Psi_{1} \sin \eta_{1} \hat{u}_{y}{ }^{\prime}+\cos \Psi_{1} \hat{u}_{z}{ }^{\prime}$,

Using equation 15 of part I and Eq. (5) above, we then obtain

$$
\begin{aligned}
\tan \eta_{1} & =\frac{\frac{a b}{A B} q_{1}{ }^{\prime} \sin \xi_{1}{ }^{\prime}}{\frac{a b}{A^{2}} q_{1}{ }^{\prime} \cos \xi_{1}{ }^{\prime}-w}, \\
\tan ^{2} \Psi_{1} & =\left(\frac{a b}{A^{2}} q_{1}{ }^{\prime} \cos \xi_{1}{ }^{\prime}-w\right)^{2}+\left(\frac{a b}{A B} q_{1}{ }^{\prime} \sin \xi_{1}{ }^{\prime}\right)^{2} \\
q_{1}{ }^{\prime} & =\frac{r_{1}{ }^{\prime}}{\left(1-{r_{1}}^{\prime 2}\right)^{1 / 2}} .
\end{aligned}
$$

We next determine the angles of incidence and refraction of the ray at its point of exit. The incidentunit wave vector at the point of exit is

$$
\hat{k}_{i 1}=\hat{k}_{t 0} \text {. }
$$

Its explicit form is given by Eqs. (15) and (19). The angle of incidence $\theta_{i 1}$ for exit on the upper surface is given by

$$
\begin{aligned}
\cos \theta_{i 1} & =\hat{n}_{1}{ }^{\prime} \cdot \hat{k}_{i 1} \\
& =\cos \Psi_{01} \cos \Psi_{1}-\sin \Psi_{01} \sin \Psi_{1} \cos \left(\eta_{0}-\eta_{1}\right) .
\end{aligned}
$$


The transmitted angle $\theta_{t 1}$ is again given by Snell's law

$$
\sin \theta_{t 1}=n \sin \theta_{i 1} .
$$

If $n \sin \theta_{i 1}>1$, the ray is incident upon this interface past the critical angle for total internal reflection and transmission does not occur in ray theory. In actuality, light waves are transmitted and are described by a Fock transition. This effect is not modeled in this paper.

For $n \sin \theta_{i 1} \leq 1$, the ray is transmitted out of the spheroid and its final outgoing unit wave vector is

$$
\begin{aligned}
\hat{k}_{t 1}= & \sin \Theta \cos (\Phi-\phi) \hat{u}_{x}{ }^{\prime}+\sin \Theta \sin (\Phi-\phi) \hat{u}_{y}{ }^{\prime} \\
& +\cos \Theta \hat{u}_{z}{ }^{\prime},
\end{aligned}
$$

which may also be written as

$$
\hat{k}_{t 1}=n \hat{k}_{i 1}+\left(\cos \theta_{t 1}-n \cos \theta_{i 1}\right) \hat{n}_{1}{ }^{\prime} .
$$

Comparing Eqs. (34) and (35), we find that the scattering angles $\Theta, \Phi$ are given by

$\cos \theta$

$$
=n \cos \Psi_{01}+\left(\cos \theta_{t 1}-n \cos \theta_{i 1}\right) \cos \Psi_{1},
$$

$\tan \Phi$

$$
=\frac{\left(\cos \theta_{t 1}-n \cos \theta_{i 1}\right) \sin \Psi_{1} \sin \eta_{1}-n \sin \Psi_{01} \sin \eta_{0}}{\left(\cos \theta_{t 1}-n \cos \theta_{i 1}\right) \sin \Psi_{1} \cos \eta_{1}-n \sin \Psi_{01} \cos \eta_{0}} .
$$

Thus given the coordinates $r_{0}{ }^{\prime}$ and $\xi_{0}{ }^{\prime}$ of an incident ray, its scattering angles $\Theta$ and $\Phi$ are obtained by combining Eqs. (12)-(14), (18) and (19), (21), (23) and (24), (28)-(30), (32) and (33), and (36) and (37).

If the transmitted ray exits on the lower or lit side of the spheroid, the unit normal $\hat{m}_{1}{ }^{\prime}$ is taken to have the spherical angles $\pi-\Psi_{1}, \eta_{1}$ with respect to the $x^{\prime} y^{\prime} z^{\prime}$ axes. For this case, the factors of $-w$ in Eqs. (28) and (29) should be replaced by $+w$, and the factors of $\cos \Psi_{1}$ in Eqs. (32) and (36) should be replaced by $-\cos \Psi_{1}$.

These expressions for the scattering angles are complicated because of the cumbersomeness of the spheroid geometry and the cumbersomeness of the geometry of refraction, and because the orientation of the spheroid is arbitrary. For arbitrary incidence it is easily shown that the planes of incidence at the ray's point of entrance into and exit from the spheroid do not coincide. Specifically, the first plane of incidence makes an angles $\eta_{0}$ with respect to the $x^{\prime}$ axis. But the second surface normal $\hat{n}_{1}{ }^{\prime}$ makes an angle $\eta_{1}$ with respect to the $x^{\prime}$ axis. If $\eta_{0} \neq \eta_{1}$, the two planes of incidence are not coincident.

If $\eta_{0}=\eta_{1}$, the two planes coincide and the entire trajectory of any given ray lies in a single plane. Comparing Eqs. (12), (24), and (28), one can see that this occurs only for $w=0$ and $A=B$ corresponding to end-on incidence, where the $z^{\prime \prime}$ spheroid major axis lies in the propagation direction of the incident plane wave. For the end-on geometry

$$
\xi_{0}{ }^{\prime}=\eta_{0}=\xi_{1}{ }^{\prime}=\eta_{1}=\Phi-\phi-\pi .
$$

Equation(13) then simplifies to

$$
\tan \Psi_{0}=\frac{b}{a} q_{0}{ }^{\prime},
$$

Eq. (21) simplifies to

$$
s_{01}=2 b\left(1-r_{0}{ }^{2}\right)^{1 / 2}\left\{\frac{\cos \Psi_{01}+\frac{b}{a} q_{0}{ }^{\prime} \sin \Psi_{01}}{\cos ^{2} \Psi_{01}+\frac{b^{2}}{a^{2}} \sin ^{2} \Psi_{01}}\right\},
$$

Eq. (23) simplifies to

$$
\begin{aligned}
r_{1}{ }^{\prime} & =\left[r_{0}{ }^{\prime 2}-\frac{2 r_{0}{ }^{\prime} s_{01}}{a} \sin \Psi_{01}+\frac{s_{01}{ }^{2}}{a^{2}} \sin ^{2} \Psi_{01}\right]^{1 / 2} \\
& =\left|r_{0}{ }^{\prime}-\frac{s_{01}}{a} \sin \Psi_{01}\right|,
\end{aligned}
$$

Eq. (29) simplifies to

$$
\tan \Psi_{1}=\frac{b}{a} q^{\prime},
$$

Eq. (32) simplifies to

$$
\theta_{i 1}=\Psi_{01}+\Psi_{1}
$$

and Eq. (36) simplifies to

$$
\Theta=\theta_{t 1}-\Psi_{1} .
$$

Unfortunately, Eqs. (19) and (39)-(44) are still sufficiently complicated that they cannot be analytically inverted to obtain $r_{0}{ }^{\prime}$ as a function of $\Theta$.

The inability to obtain $r_{0}{ }^{\prime}$ and $\xi_{0}{ }^{\prime}$ as functions of $\Theta$ and $\Phi$ prevents us from analytically evaluating the magnitude of the transmitted electric field,

$$
S_{\text {trans }}(\Theta, \Phi)=\left(\frac{k^{2} A B r_{0}{ }^{1 / 2}}{\Delta \sin \Theta}\right)^{1}
$$

in terms of $\Theta$ and $\Phi$ alone, where

$$
\Delta=\left|\begin{array}{ll}
\frac{\partial \Theta}{\partial r_{0}{ }^{\prime}} & \frac{\partial \Theta}{\partial \xi_{0}{ }^{\prime}} \\
\frac{\partial \Phi}{\partial r_{0}{ }^{\prime}} & \frac{\partial \Phi}{\partial \xi_{0}{ }^{\prime}}
\end{array}\right|
$$

(See equations 17 and 18 of part I). In Section 4 the 
numerical evaluation of Eqs. (45) and (46) is discussed.

\section{B. Phase of the Transmitted Electric Field}

The trajectories of both the transmitted ray and the reference ray are shown in Fig. 2. The reference ray is described in detail in part I. Briefly, it propagates along the $z$ axis to the origin as if the spheroid were absent. It then turns to the $\Theta, \Phi$ direction and propagates in that direction to the far zone. The distance $\alpha_{R}$ from the spheroid entrance plane UU' to the origin is

$$
\alpha_{R}=z_{\text {min }}{ }^{\prime},
$$

where $z_{\min }{ }^{\prime}$ is the lowest point on the spheroid surface. The distance from the origin to the spheroid exit plane $\mathrm{VV}^{\prime}$ is (equation 41 of part I)

$$
\begin{aligned}
\beta_{R}= & \left\{\frac{A^{2}}{a^{2} b^{2}}[\cos \Theta-w \sin \Theta \cos (\Phi-\phi)]^{2}\right. \\
& \left.+\sin ^{2} \Theta\left[\frac{\cos ^{2}(\Phi-\phi)}{A^{2}}+\frac{\sin ^{2}(\Phi-\phi)}{B^{2}}\right]\right\}^{-1 / 2} .
\end{aligned}
$$

The optical path length of the transmitted ray between the spheroid entrance and exit planes is $\alpha_{0}+n s_{01}+\beta_{1}$, where $\alpha_{0}$ is the distance from the entrance plane to the ray's point of entrance on the spheroid's lit surface and $\beta_{1}$ is the distance from the ray's point of exit on either the spheroid's shadowed or lit surface to the exit plane. From Fig. 2, the distance $\alpha_{0}$ is given by

$$
\alpha_{0}=\alpha_{R}+z_{0}{ }^{\prime}\left(r_{0}{ }^{\prime}, \xi_{0}{ }^{\prime}\right) .
$$

The distance $\beta_{1}$ is determined as it was for reflection in part I. The equation of the outgoing reference

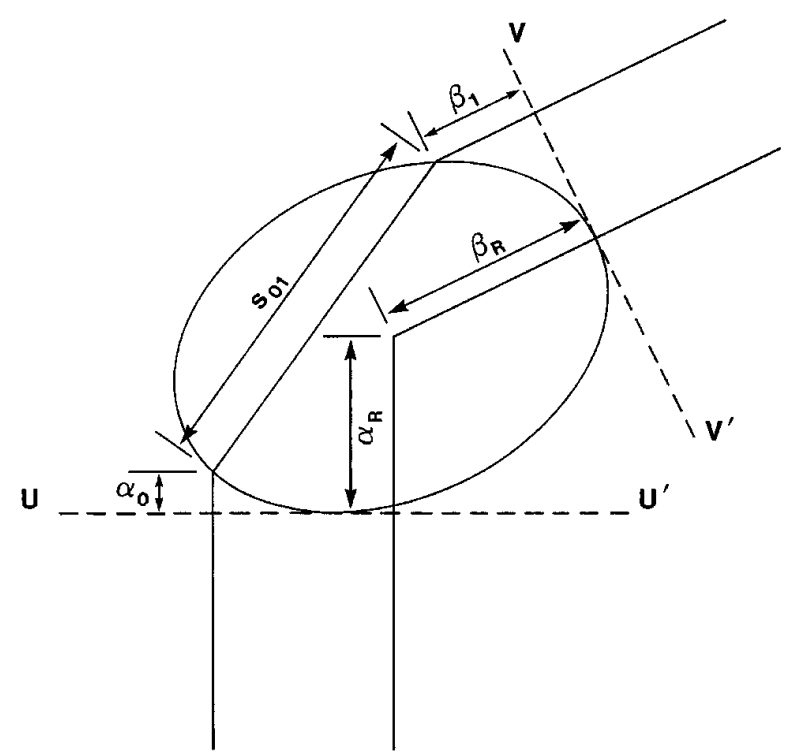

Fig. 2. Trajectories of the transmitted ray and the reference ray. The spheroid entrance plane is $\mathrm{UU}^{\prime}$, and the spheroid exit plane is $\mathrm{VV}^{\prime}$. ray is

$$
\begin{aligned}
& x^{\prime}=\beta_{R} \sin \Theta \cos (\Phi-\phi), \\
& y^{\prime}=\beta_{R} \sin \Theta \sin (\Phi-\phi), \\
& z^{\prime}=\beta_{R} \cos \Theta
\end{aligned}
$$

for $\beta_{R} \geq 0$. The equation of the plane normal to this line at the point $\beta_{R}$ given by Eq. (48) may now be constructed. This is the exit plane VV' of Fig. 2. The intersection of this plane with the outgoing transmitted ray

$$
\begin{aligned}
& x^{\prime}=A r_{1}{ }^{\prime} \cos \xi_{1}{ }^{\prime}+\beta_{1} \sin \Theta \cos (\Phi-\phi), \\
& y^{\prime}=B r_{1}{ }^{\prime} \sin \xi_{1}{ }^{\prime}+\beta_{1} \sin \Theta \sin (\Phi-\phi), \\
& z^{\prime}=z_{1}{ }^{\prime}\left(r_{1}{ }^{\prime}, \xi_{1}{ }^{\prime}\right)+\beta_{1} \cos \Theta
\end{aligned}
$$

for $\beta_{1} \geq 0$ is then determined, giving

$$
\begin{aligned}
\beta_{1}= & \beta_{R}-A r_{1}{ }^{\prime} \cos \xi_{1}{ }^{\prime} \sin \Theta \cos (\Phi-\phi) \\
& -B r_{1}{ }^{\prime} \sin \xi_{1}{ }^{\prime} \sin \Theta \sin (\Phi-\phi)-z_{1}{ }^{\prime} \cos \Theta .
\end{aligned}
$$

The optical path length of the transmitted ray with respect to that of the reference ray is then

$$
\begin{aligned}
L_{\text {trans }}= & \alpha_{0}+n s_{01}+\beta_{1}-\alpha_{R}-\beta_{R}=z_{0}{ }^{\prime}+n s_{01} \\
& -A r_{1}{ }^{\prime} \cos \xi_{1}{ }^{\prime} \sin \Theta \cos (\Phi-\phi)-B r_{1}{ }^{\prime} \\
& \times \sin \xi_{1}{ }^{\prime} \sin \Theta \sin (\Phi-\phi)-z_{1}{ }^{\prime} \cos \Theta
\end{aligned}
$$

independent of whether $z_{1}{ }^{\prime}$ is on the shadowed or lit side of the spheroid.

Each ray transmitted through a spherical particle participates in two focusing caustics ${ }^{32-34}$ : (i) a spherical aberration cusp of revolution, which is also known as the tangential caustic, and (ii) an axial spike caustic on the cusp axis, which is also known as the sagittal caustic. The cusp of revolution has its apex at the paraxial focal point and it extends through the sphere surface to its interior. ${ }^{34}$ The axial spike caustic meets the cusp of revolution at the paraxial focal point. Using van de Hulst's rule for equating the number of $-\pi / 2$ jumps in the phase of a ray to the number of caustic participations ${ }^{35}$ and including another factor of $-\pi / 2$ to compensate for the overall factor of $i$ in Eq. (3), we obtain

$$
\delta_{\text {trans }}=\frac{2 \pi}{\lambda} L_{\text {trans }}-\frac{3 \pi}{2}
$$

as the phase of the transmitted ray.

For an arbitrary spheroid eccentricity and orientation the situation is considerably more complicated. Nye's analysis of the one-internal-reflection rays for side-on incidence ${ }^{19}$ gives a hint as to the complexity of the focusing caustics of spheriods. We have not yet fully solved the caustic problem for transmission through an arbitrarily oriented spheroid. But in the remainder of this section the complete solution for end-on incidence is given first and then a few comments concerning the case of arbitrary orienta- 
tion are made. In Fig. 3 we show the evolution of the transmission caustics as $b / a$ is increased from 1.0 for the specific example of $n=1.333$. Figure 3(a) corresponds to transmission through a sphere, where the cusp caustic of revolution points outward from the sphere. Figure $3(b)$ corresponds to $b / a=1.33$. The cusp caustic begins to retract into the spheroid while continuing to point outward. Figure 3(c) corresponds to

$$
\frac{b^{2}}{a^{2}}=\frac{n^{2}}{n^{2}-1},
$$

where all the incident rays focus at the point ${ }^{36,37}$

$$
z^{\prime}=\left(b^{2}-a^{2}\right)^{1 / 2},
$$

independent of $r_{0}{ }^{\prime}$ and $\xi_{0}{ }^{\prime}$. In Figs. $3(\mathrm{~d})-3(\mathrm{~h})$ the interior cusp caustic of revolution points inward toward the center of the spheroid. Thus the sequence in Figs. 3(a)-3(d) may be interpreted as turning the cusp of revolution inside out through its contraction to a point focus in Fig. 3(c).

Figure $3(\mathrm{e})$ corresponds to ${ }^{38}$

$$
\frac{b^{2}}{a^{2}}=\frac{n}{n-1},
$$

where the paraxial rays cross the $z^{\prime}$ axis at the origin and exit the spheroid with the scattering angle $\Theta=$ $0^{\circ}$, giving a forward glory. The forward glory is a third focusing caustic for this case. For $b^{2} / a^{2}$ only slightly larger than $n /(n-1)$, the rays with small $r_{0}{ }^{\prime}$ exit the spheroid with a negative scattering angle. As $r_{0}{ }^{\prime}$ is increased, the scattering angle further decreases, reaches a relative minimum, ${ }^{38}$ and then increases, reaching $\Theta=0^{\circ}$ when

$$
q_{0}{ }^{\prime 2}=\frac{n^{2}\left(\frac{b^{2}}{a^{2}}-1\right)^{2}}{b^{2} / a^{2}}-\frac{b^{2}}{a^{2}} .
$$

For yet larger values of $r_{0}{ }^{\prime}$, the scattering angle then becomes positive. The rays between $r_{0}{ }^{\prime}=0$ and the value of $r_{0}{ }^{\prime}$ corresponding to the relative minimum of $\Theta$ (i.e., $r_{0}{ }^{\prime m i n}$ ) form a third and fourth focusing caustic. These caustics are a new cusp of revolution far outside the spheroid and pointing inward and a new axial spike caustic. The new cusp caustic evolves into a far-zone transmission rainbow ${ }^{38}$ at the minimum scattering angle $\Theta_{\min }$ corresponding to $r_{0}{ }^{\prime m i n}$. For $r_{0}{ }^{\prime}$ between $r_{0}{ }^{\prime}$ min and the value implicitly given in Eq. (58) only the new axial spike caustic is formed. It extends from beyond the second cusp point out to infinity, where it evolves into the forward glory.

As $b^{2} / a^{2}$ is further increased, the new inwardpointing cusp also begins to turn itself inside out by progressing through a butterfly of revolution caustic, ${ }^{39}$ as is shown in Fig. $3(\mathrm{f})$ and greatly magnified in Fig. $3(\mathrm{~g})$. Finally, if $b^{2} / a^{2}$ is sufficiently large, the evolution of the butterfly caustic reaches completion, and it becomes an outward-pointing cusp of revolution, as in Fig. 3(h). Although Figs. 3(a) $-3(\mathrm{~h})$ describe $n=1.333$, the same evolution of the caustics was observed for all the other refractive indices examined for the end-on spheroid geometry.

As a result, depending on the values of $n, b^{2} / a^{2}$, and $r_{0}{ }^{\prime}$ for end-on incidence, a transmitted ray participates in either 2,3 , or 4 focusing caustics and acquires a transmitted phase of either $-3 \pi / 2,-2 \pi$, or $-5 \pi / 2$, respectively [including an additional factor of $-\pi / 2$ to compensate for the overall factor of $i$ in Eq. (3)]. The number of caustic participations is illustrated in the phase diagram of Fig. 4. The case of an oblate end-on spheroid presents no special problem because the rays transmitted through it resemble the rays transmitted through a thick lens. Each ray participates in only 2 caustics, a spherical aberration cusp of revolution and its associated axial spike caustic. ${ }^{33}$

If the $z^{\prime \prime}$ axis of the spheroid is now tilted with respect to the lab $z$ axis for $b^{2} / a^{2}<n /(n-1)$, the rotational symmetry that produced the degeneracy of the axial spike caustic is lost and it deforms into a four-cusped astroid caustic. ${ }^{40}$ The cusp caustic also loses its rotational symmetry. ${ }^{41}$ The combination of the distorted cusp and astroid has an astigmatic focusing character, and sections through the composite caustic resemble the evolution shown in appendix 2 of Ref. 39. For $b^{2} / a^{2} \gtrsim n /(n-1)$ the new cusp and axial spike caustics deform into a second composite astigmatic focusing caustic as in appendix 2 of Ref. 39. For yet larger values of $b / a$, the butterfly of revolution caustic evolves into a complicated structure that we do not yet fully understand. But in any event, as long as the spheroid eccentricity satisfies $b^{2} / a^{2}<n /(n-1)$, each ray participates in two focusing caustics, and the transmitted phase shift for an arbitrary spheroid orientation is given by Eq. (54).

\section{Polarization of the Transmitted Electric Field}

The incident plane wave is polarized so that its electric field makes an angle $\chi$ with the $x$ axis. The polarization vector of the incident plane wave with respect to the $x^{\prime} y^{\prime} z^{\prime}$ rotated lab axes is then (equation 48 of part I)

$$
\hat{\epsilon}_{\text {inc }}=\cos (\chi-\phi) \hat{u}_{x}{ }^{\prime}+\sin (\chi-\phi) \hat{u}_{y}{ }^{\prime} .
$$

In part I, the unit vectors in the $\mathrm{TE}$ and $\mathrm{TM}$ polarization directions were taken to be (equations 49 and 50 of part I)

$$
\begin{aligned}
& \widehat{\mathrm{TE}}_{\mathrm{inc}}=\frac{\hat{m}^{\prime} \times \hat{k}_{\mathrm{inc}}}{\sin \theta_{\mathrm{inc}}} \\
& \widehat{\mathrm{TE}}_{\mathrm{inc}}=\frac{\hat{k}_{\mathrm{inc}} \times\left(\hat{m}^{\prime} \times \hat{k}_{\mathrm{inc}}\right)}{\sin \theta_{\mathrm{inc}}} .
\end{aligned}
$$

When considering the transmitted ray we define the $\mathrm{TE}$ and TM polarization unit vectors to be the negative of Eq. (60). The reason for this is as 


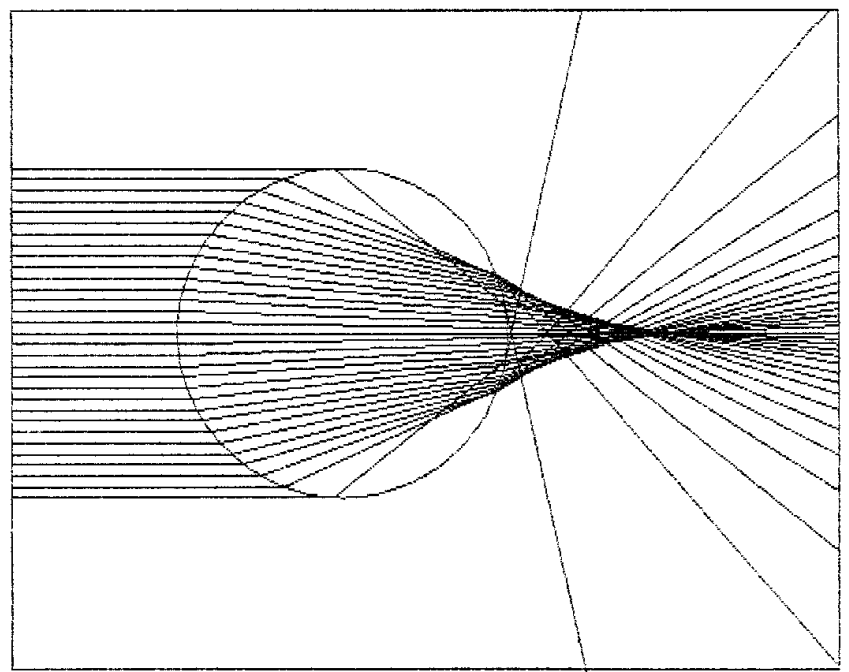

(a)

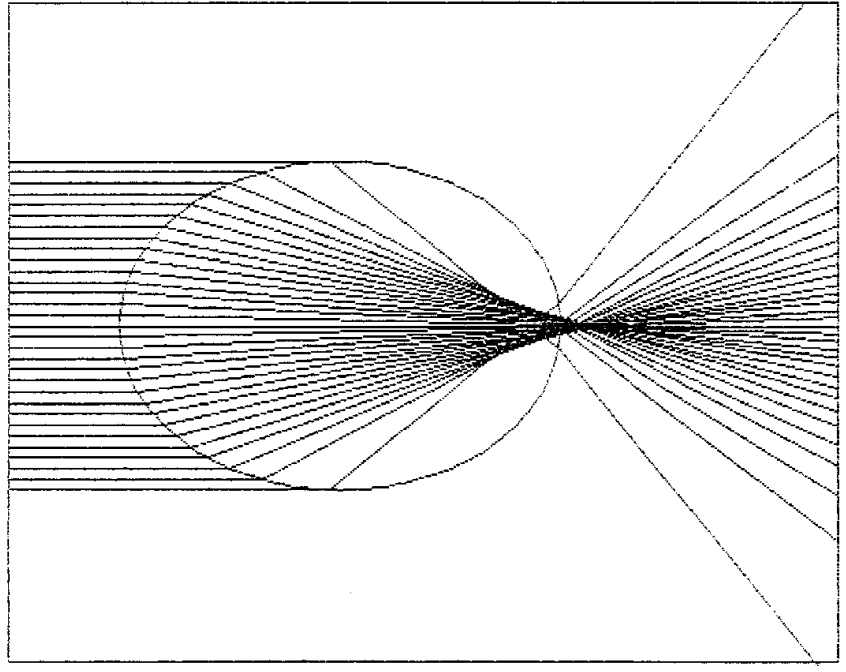

(b)

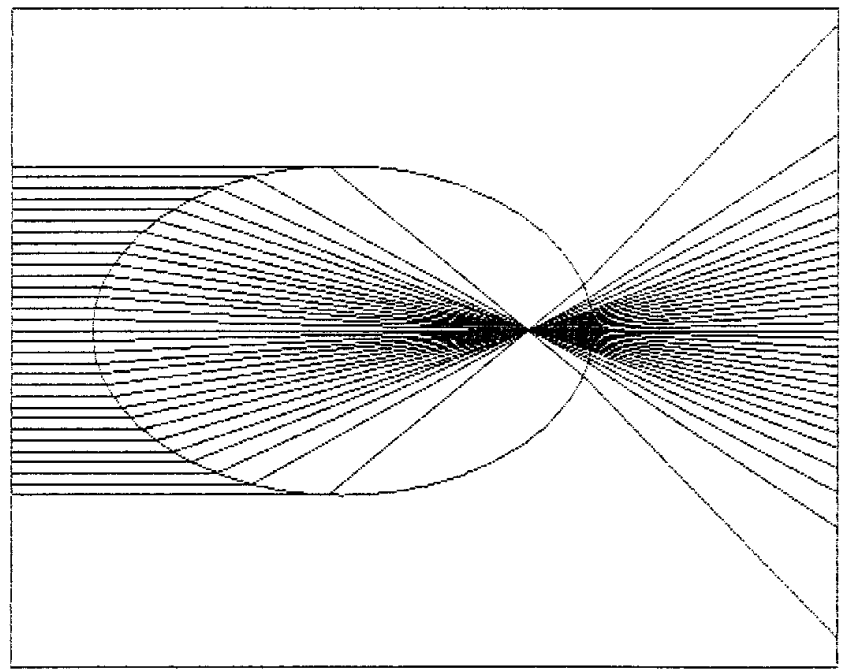

(c)

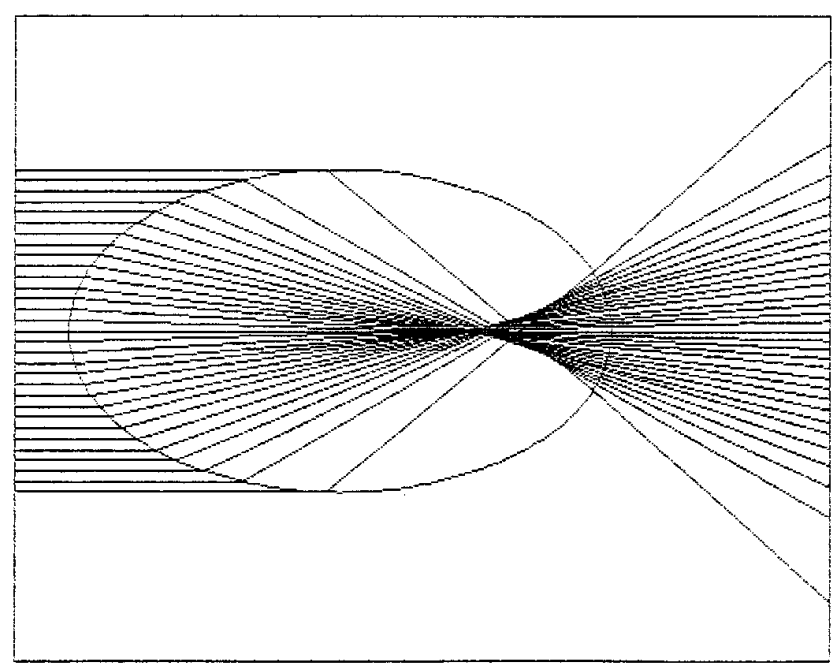

(d)

Fig. 3 (continued)

$$
\begin{aligned}
& \widehat{\mathrm{TE}}_{t 0}=\frac{\hat{k}_{t 0} \times \hat{m}_{0}^{\prime}}{\sin \theta_{t 0}}, \\
& \widehat{\mathrm{TM}}_{t 0}=\frac{\hat{k}_{t 0} \times\left(\hat{k}_{t 0} \times \hat{m}_{0}{ }^{\prime}\right)}{\sin \theta_{t 0}}, \\
& \widehat{\mathrm{TE}}_{i 1}=\frac{\hat{k}_{i 1} \times \hat{n}_{1}^{\prime}}{\sin \theta_{i 1}}, \\
& \widehat{\mathrm{TM}}_{i 1}=\frac{\hat{k}_{i 1} \times\left(\hat{k}_{i 1} \times \hat{n}_{1}{ }^{\prime}\right)}{\sin \theta_{i 1}} \\
& \widehat{\mathrm{TE}}_{t 1}=\frac{\hat{k}_{t 1} \times \hat{n}_{1}{ }^{\prime}}{\sin \theta_{t 1}} \\
& \widehat{\mathrm{TM}}_{t 1}=\frac{\hat{k}_{t 1} \times\left(\hat{k}_{t 1} \times \hat{n}_{1}^{\prime}\right)}{\sin \theta_{t 1}}
\end{aligned}
$$

as is shown in Fig. 5. 


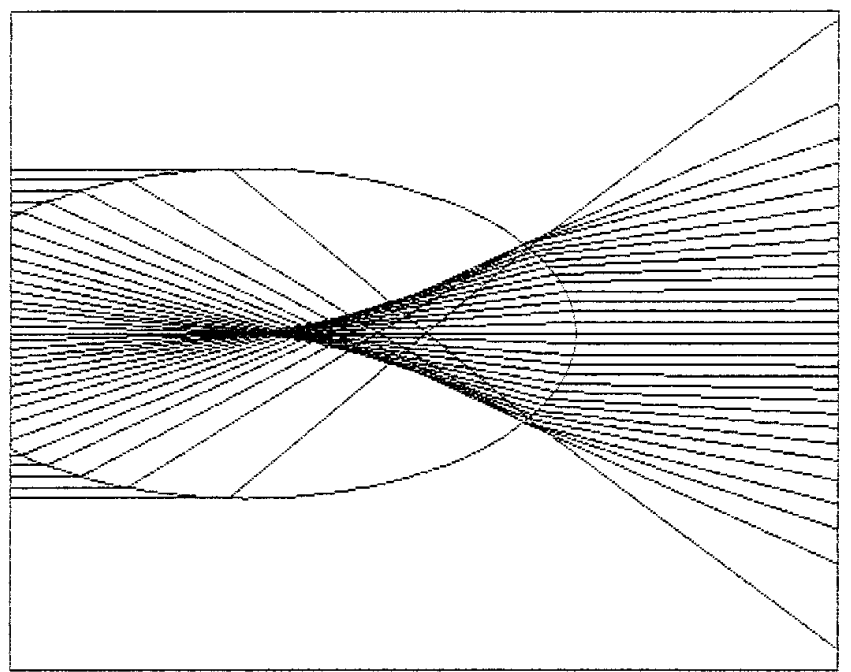

(e)

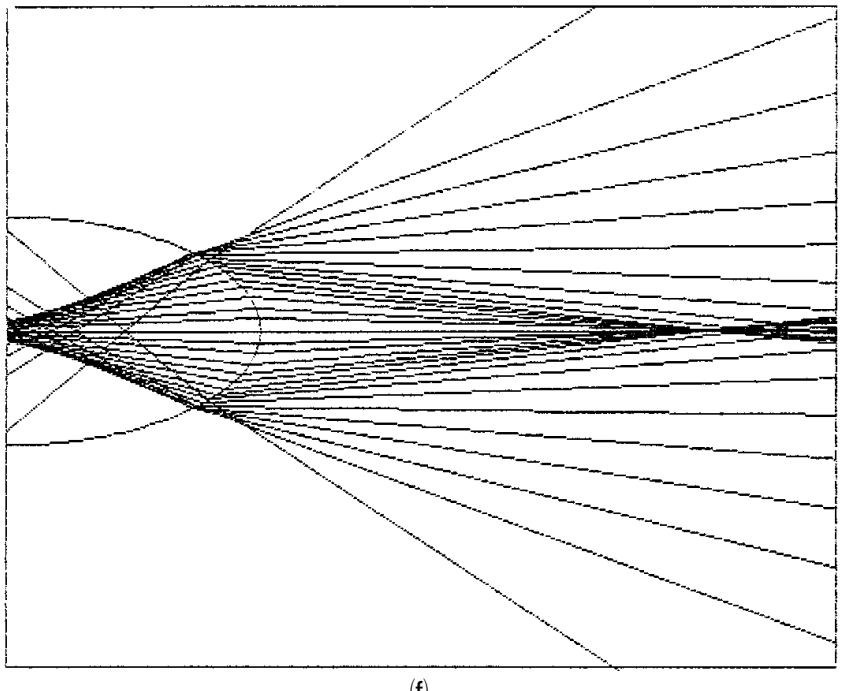

(f)

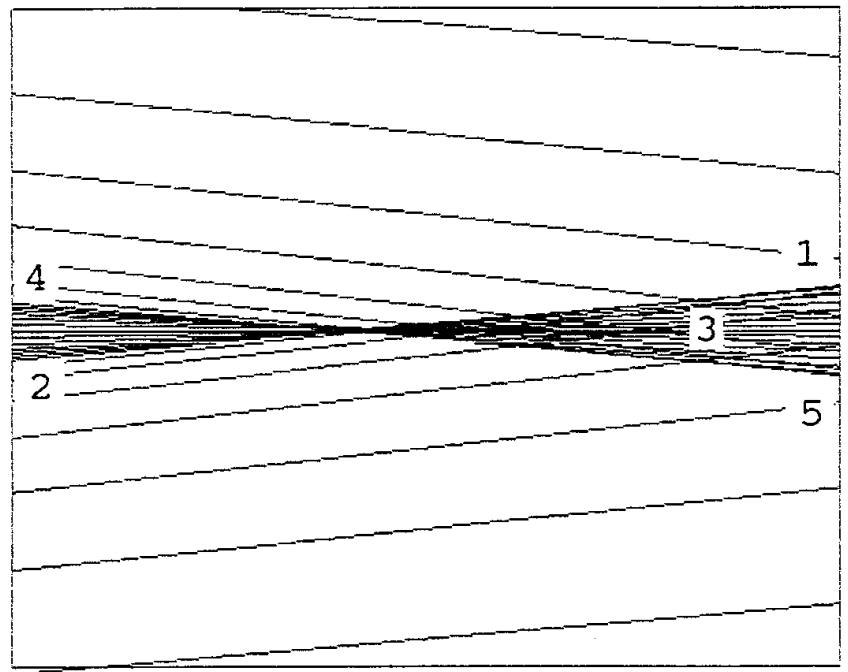

(g)

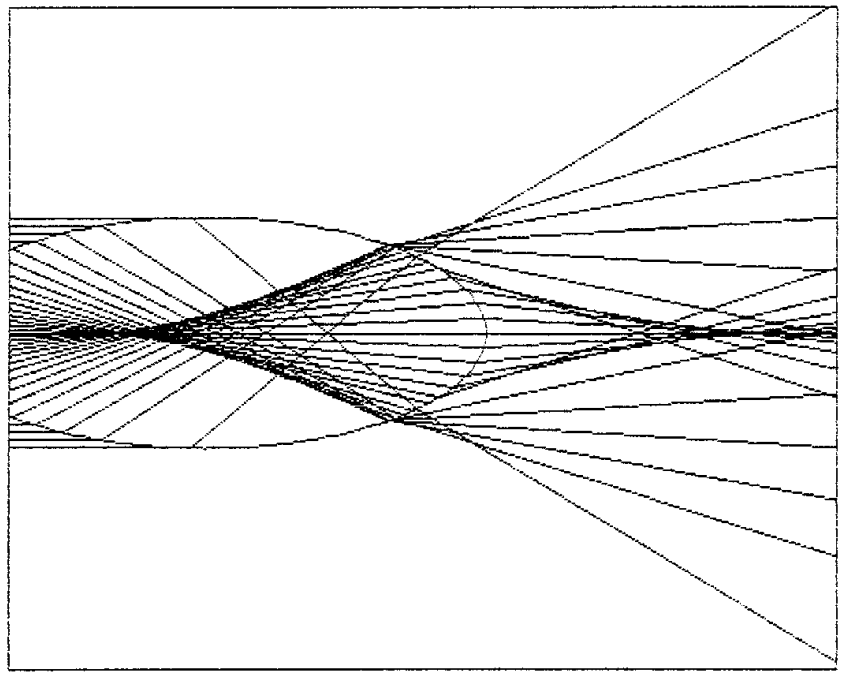

(h)

Fig. 3. Rays transmitted through a spheroid for $n=1.333$. (a) $b / a=1$ corresponding to a sphere. The caustics are a cusp of revolution pointing outward and an axial spike caustic. (b) $b / a=1.33$. The cusp starts to retract into the spheroid. (c) $b / a=1.5123$. All the rays focus at a single point. (d) $b / a=1.67$. The caustics are a cusp of revolution pointing inward and an axial spike caustic. (e) $b / a=$ 2.0. Paraxial rays have a scattering angle of $\Theta=0^{\circ}$ corresponding to a forward glory. (f) $b / a=2.25$. The paraxial rays form a butterfly of revolution caustic and a second axial spike caustic. (g) Butterfly caustic of (f) magnified by a factor of 11.67 . The caustic begins at location 1 and then continues in order to the locations $2,3,4,5$. (h) $b / a=2.45$. The second-cusp caustic points outward. As a function of $r_{0}{ }^{\prime}$ there are two critical angles for total internal reflection.

For scattering by a spheroid, the rays reflected and transmitted in the $\Theta, \Phi$ direction again in general originate on opposite ends of the lit side of the spheroid. As a result, we use the TE and TM polarization directions of Eqs. (61)-(64) for spheroid scattering as well. For an arbitrary spheroid orientation with $\eta_{0} \neq \eta_{1}$, the two TE unit vectors $\widehat{\mathrm{TE}}_{t 0}$ and $\widehat{\mathrm{TE}}_{i 1}$, which describe the ray inside the spheroid referenced with respect to the 0 interface and the 1 interface, respectively, do not coincide. This is the source of the first of the cross-polarization effects in the transmitted intensity. We derive this effect as follows. The incident-ray polarization vector of Eq. (59) may be decomposed into the TE and TM compo- nents of Eq. (61), giving

$$
\hat{\epsilon}_{\text {inc }}=\cos \gamma_{0} \widehat{\mathrm{TE}}_{i 0}+\sin \gamma_{0} \widehat{\mathrm{TM}}_{i 0},
$$

where

$$
\gamma_{0}=\chi-\phi-\eta_{0}-\frac{\pi}{2}
$$

After transmission into the spheroid, the polarization vector of the ray becomes

$$
\boldsymbol{\epsilon}_{t 0}=t_{\mathrm{TE}}{ }^{0} \cos \gamma_{0} \widehat{\mathrm{TE}}_{t 0}+t_{\mathrm{TM}}{ }^{0} \sin \gamma_{0} \widehat{\mathrm{TM}}_{t 0}
$$




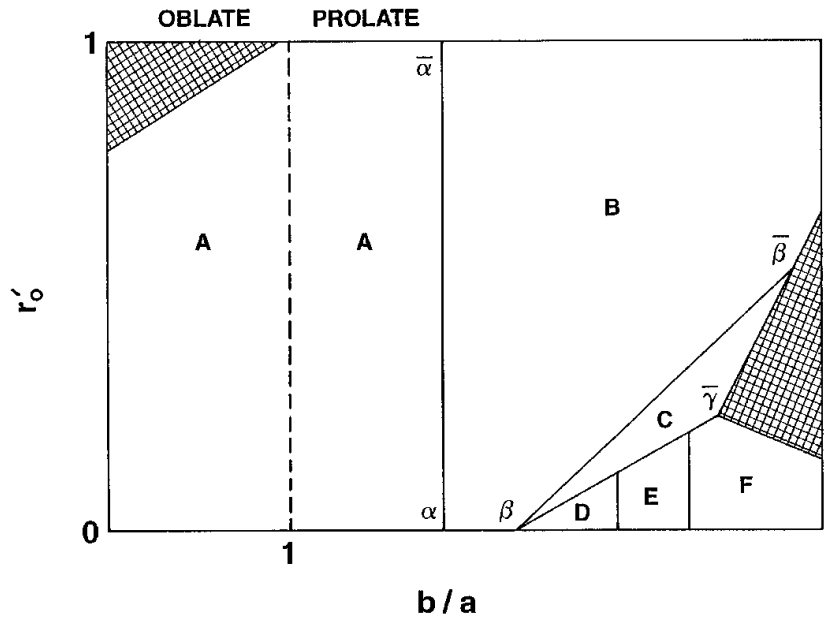

Fig. 4. Phase-space diagram for the caustics produced by the transmitted rays when $r_{0}{ }^{\prime}$ and $b / a$ are varied while the refractive index $n$ is held fixed. In region A there are two caustics, a cusp of revolution pointing outward and an axial spike. Along the line $\alpha \bar{\alpha}$ corresponding to Eq. (55) these caustics contract to a point focus. In region B there are two caustics, a cusp of revolution pointing inward and an axial spike. In region $\mathrm{C}$ there are three caustics, a cusp of revolution pointing inward and two axial spikes. In regions $\mathrm{D}, \mathrm{E}, \mathrm{F}$, there are four caustics. In $\mathrm{D}$ they are two cusps pointing inward and two axial spikes. In $\mathrm{E}$ they are an inward-pointing cusp, a butterfly, and two axial spikes. In F they are an inward-pointing and an outward-pointing cusp, and two axial spikes. The line $\beta \bar{\beta}$ corresponds to the forward glory given by Eq. (58), and the line $\beta \bar{\gamma}$ is the transmission rainbow. The cross-hatched regions denote the absence of transmitted rays as a result of total internal reflection as in Eq. (33).

where $t_{\mathrm{TE}}{ }^{0}\left(\theta_{i 0}\right)$ and $t_{\mathrm{TM}}{ }^{0}\left(\theta_{i 0}\right)$ are the Fresnel coefficients for transmission corresponding to the angle of incidence $\theta_{i 0}$. As mentioned above, the $\widehat{\mathrm{TE}}_{t 0}, \widehat{\mathrm{TM}}_{t 0}$ and the $\widehat{\mathrm{TE}}_{i 1}, \widehat{\mathrm{TM}}_{i 1}$ polarization vectors are rotated with respect to each other because the 0 -interface and the 1-interface planes of incidence do not coincide. Specifically, by substituting Eqs. (11), (15), and (27) into Eqs. (62) and (63) we obtain

$$
\begin{aligned}
& \widehat{\mathrm{TE}}_{t 0}=\cos \Delta_{01} \widehat{\mathrm{TE}}_{i 1}+\sin \Delta_{01} \widehat{\mathrm{TM}}_{i 1}, \\
& \widehat{\mathrm{TM}}_{t 0}=-\sin \Delta_{01} \widehat{\mathrm{TE}}_{i 1}+\cos \Delta_{01} \widehat{\mathrm{TM}}_{i 1},
\end{aligned}
$$

where

$$
\tan \Delta_{01}=\frac{\sin \Psi_{1} \sin \left(\eta_{0}-\eta_{1}\right)}{\sin \Psi_{10} \cos \Psi_{1}+\cos \Psi_{10} \sin \Psi_{1} \cos \left(\eta_{0}-\eta_{1}\right)}
$$

if the ray exits the spheroid on its shadowed side. If it exits on the lit side, the factor of $\cos \Psi_{1}$ in Eq. (69) is replaced by $-\cos \Psi_{1}$. The polarization vector of the ray incident at the point of exit on either the shadowed or lit side of the spheroid is then

$$
\begin{aligned}
\boldsymbol{\epsilon}_{i 1}= & \left.t_{\mathrm{TE}}^{0} \cos \gamma_{0} \cos \Delta_{01}-t_{\mathrm{TM}^{0}} \sin \gamma_{0} \sin \Delta_{01}\right) \widehat{\mathrm{TE}}_{i 1} \\
& +\left(t_{\mathrm{TE}}{ }^{0} \cos \gamma_{0} \sin \Delta_{01}+t_{\mathrm{TM}}{ }^{0} \sin \gamma_{0} \cos \Delta_{01}\right) \widehat{\mathrm{TM}}_{i 1} .
\end{aligned}
$$

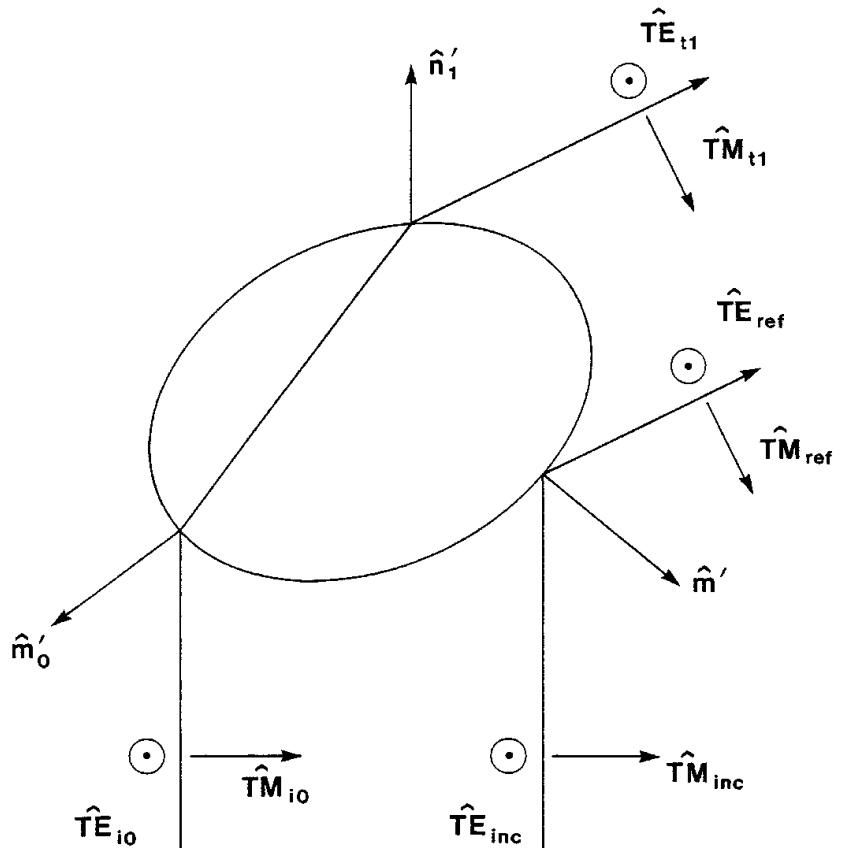

Fig. 5. TE and TM polarization directions for the reflected ray and the transmitted ray of Eq. (60) and Eqs. (61)-(64), respectively.

Finally, the polarization vector of the outgoing ray after it exits the spheroid is

$$
\begin{aligned}
\boldsymbol{\epsilon}_{t 1}= & \left(t_{\mathrm{TE}}{ }^{0} t_{\mathrm{TE}}{ }^{1} \cos \gamma_{0} \cos \Delta_{01}-t_{\mathrm{TM}}^{0} t_{\mathrm{TE}}{ }^{1} \sin \gamma_{0} \sin \Delta_{01} \widehat{\mathrm{TE}}_{t 1}\right. \\
& +\left(t_{\mathrm{TE}}{ }^{0} t_{\mathrm{TM}}{ }^{1} \cos \gamma_{0} \sin \Delta_{01}\right. \\
& \left.+t_{\mathrm{TM}}{ }^{0} t_{\mathrm{TM}}{ }^{1} \sin \gamma_{0} \cos \Delta_{01}\right) \widehat{\mathrm{TM}}_{t 1}
\end{aligned}
$$

where $t_{\mathrm{TE}}{ }^{1}\left(\theta_{i 1}\right)$ and $t_{\mathrm{TM}}{ }^{1}\left(\theta_{i 1}\right)$ are the Fresnel coefficients for transmission corresponding to the angle of incidence $\theta_{i 1}$. Again, $\theta_{i 1}$ must be less than the critical angle for total internal reflection in order for the ray to exit the spheroid.

\section{Cross-Polarization Effects}

A pictorial representation of Eq. (71) is given in Fig. 6. For scattering by a sphere, the only possibilities are TE polarization in producing TE polarization out and TM polarization in producing TM polarization out. For scattering by an arbitrarily oriented spheroid, these two possibilities are weighted by $\cos \Delta_{01}$. The cross-polarization possibilities of TE polarization in producing TM polarization out and TM polarization in producing TE polarization out are weighted by $\pm \sin \Delta_{01}$. For scattering by a spheroid with end-on incidence, $\eta_{0}=\eta_{1}$ and $\Delta_{01}=0$, which eliminates this cross-polarization effect.

The second cross-polarization effect in forwardhemisphere scattering by a transparent spheroid is due to the fact that $\widehat{\mathrm{TE}}_{\mathrm{ref}}, \widehat{\mathrm{TM}}_{\mathrm{ref}}$ of equations 54 and 55 of part I are rotated with respect to $\widehat{\mathrm{TE}}_{t 1}, \widehat{\mathrm{TM}}_{t 1}$ of Eq. (64). This may be shown as follows. Substituting for $\hat{k}_{t 1}$ and $\hat{n}_{1}^{\prime}$ in Eq. (64) and comparing with 


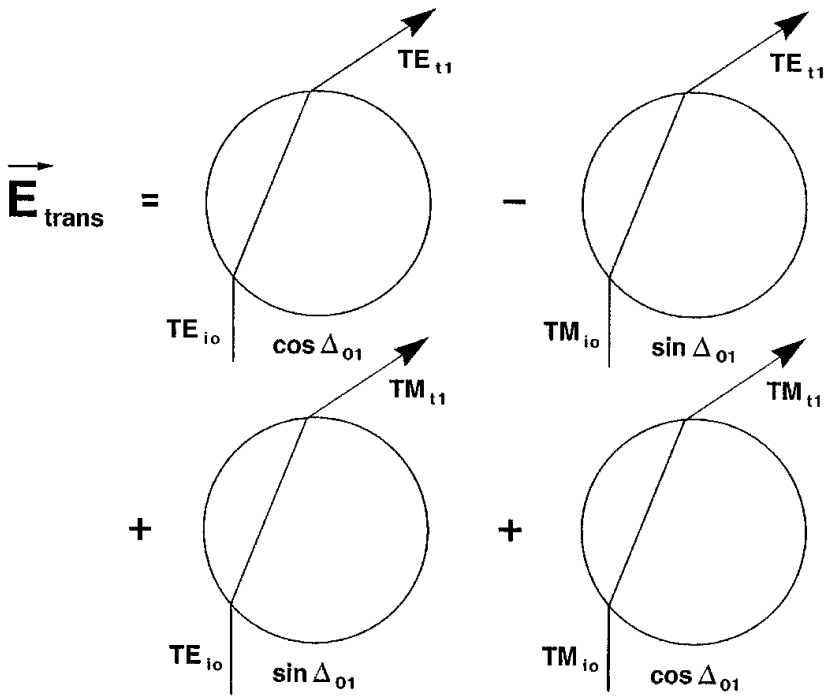

Fig. 6. Contributions to the transmitted electric field. The contributions proportional to $\cos \Delta_{01}$ are the polarization terms that describe transmission by a sphere or end-on spheroid. The contributions proportional to $\sin \Delta_{01}$ are the first type of crosspolarization terms.

equations 54 and 55 of part I gives

$$
\begin{aligned}
& \widehat{\mathrm{TE}}_{t 1}=\cos \Delta_{R 1} \widehat{\mathrm{TE}}_{\mathrm{ref}}+\sin \Delta_{R 1} \widehat{\mathrm{TM}}_{\mathrm{ref}}, \\
& \widehat{\mathrm{TM}}_{t 1}=-\sin \Delta_{R 1} \widehat{\mathrm{TE}}_{\mathrm{ref}}+\cos \Delta_{R 1} \widehat{\mathrm{TM}}_{\mathrm{ref}},
\end{aligned}
$$

where

$\tan \Delta_{R 1}$

$$
=\frac{\sin \Psi_{1} \sin \left(\Phi-\phi-\eta_{1}\right)}{\sin \Theta \cos \Psi_{1}-\cos \Theta \sin \Psi_{1} \cos \left(\Phi-\phi-\eta_{1}\right)},
$$

if the ray exits the spheroid on its shadowed side. If it exits on the lit side, the factor of $\cos \Psi_{1}$ in Eq. (73) is replaced by $-\cos \Psi_{1}$. This cross-polarization effect is pictorially represented in Fig. 7. For end-on incidence Eq. (38) gives $\Phi-\phi-\eta_{1}=\pi$ and $\Delta_{R 1}=0$, eliminating this cross-polarization effect as well.

At this point we may combine Eqs. (3) and (72) with equation 81 of part I to obtain the diffracted plus reflected plus transmitted electric field in ray theory. We relate this electric field to the amplitudescattering matrix ${ }^{42,43}$

$$
\left[\begin{array}{ll}
S_{2} & S_{3} \\
S_{4} & S_{1}
\end{array}\right]
$$

defined by

$$
\mathbf{E}_{\text {diff }+ \text { ref }+ \text { trans }}=\frac{i E_{0}}{k R} \exp (i k R-i \omega t)\left[\begin{array}{ll}
S_{2} & S_{3} \\
S_{4} & S_{1}
\end{array}\right]\left[\begin{array}{c}
\sin \gamma \\
\cos \gamma
\end{array}\right] .
$$

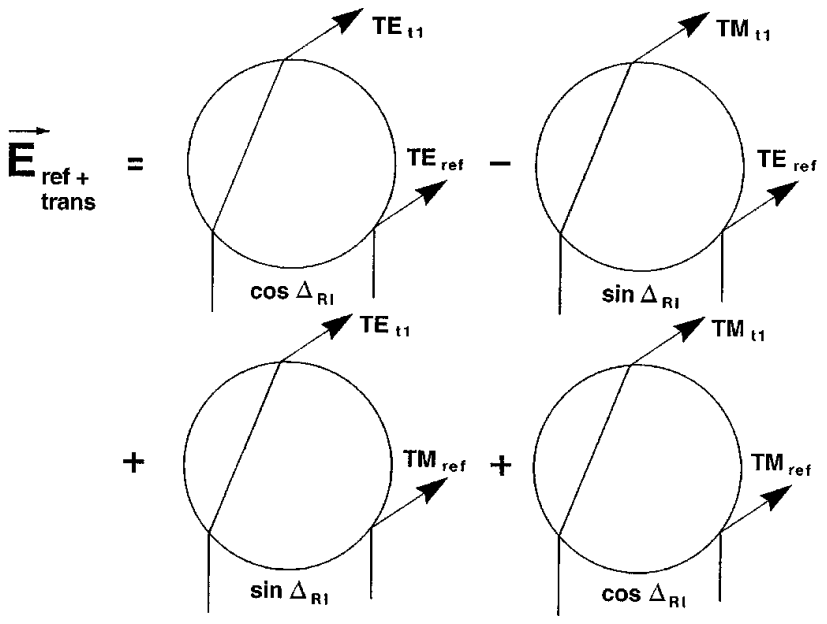

Fig. 7. Contributions to the reflected plus transmitted electric field. The contributions proportional to $\cos \Delta_{R 1}$ are the polarization terms that describe scattering by a sphere or end-on spheroid. The contributions proportional to $\sin \Delta_{R 1}$ are the second type of cross-polarization terms.

In Eq. (74) the incident-field column vector

$$
\left[\begin{array}{l}
\sin \gamma \\
\cos \gamma
\end{array}\right]
$$

is referenced with respect to the TM polarization component (i.e., sin $\gamma$ ) and the TE polarization component (i.e., $\cos \gamma$ ) of the incident ray that will be reflected into the $\Theta, \Phi$ direction. The angle $\gamma$ is given by equation 53 of part I, i.e.,

$$
\gamma=\chi-\phi-\eta-\frac{3 \pi}{2}=\chi-\Phi-\frac{3 \pi}{2} .
$$

The scattered-field column vector

$$
\left[\begin{array}{l}
S_{2} \sin \gamma+S_{3} \cos \gamma \\
S_{4} \sin \gamma+S_{1} \cos \gamma
\end{array}\right]
$$

is referenced with respect to the polarization directions $\widehat{\mathrm{TM}}_{\mathrm{ref}}$ and $\widehat{\mathrm{TE}}_{\mathrm{ref}}$. But the polarization vector of the transmitted ray was calculated in Subsection 2.C. with respect to the ray's own TE and TM polarization directions of Eqs. (63) and (64) rather than with respect to the reflected ray's TE and TM polarization directions. The transformation of the incident directions of Eqs. (60) and (61) is

$$
\begin{aligned}
& \widehat{\mathrm{TE}}_{i 0}=\cos \Omega \widehat{\mathrm{TE}}_{\mathrm{inc}}-\sin \Omega \widehat{\mathrm{TM}}_{\mathrm{inc}}, \\
& \widehat{\mathrm{TM}}_{i 0}=\sin \Omega \widehat{\mathrm{TE}}_{\mathrm{inc}}+\cos \Omega \widehat{\mathrm{TM}}_{\mathrm{inc}},
\end{aligned}
$$

where

$$
\Omega=\gamma_{0}-\gamma=\pi+\eta-\eta_{0}=\pi+\Phi-\phi-\zeta_{0},
$$

For end-on incidence, Eq. (38) gives $\Omega=0$.

The scattering matrix of Eq. (74) may then be 
written as

$$
\begin{aligned}
{\left[\begin{array}{ll}
S_{2} & S_{3} \\
S_{4} & S_{1}
\end{array}\right]=} & S_{\text {diff }}\left[\begin{array}{ll}
1 & 0 \\
0 & 1
\end{array}\right]+S_{\text {ref }} \exp \left(i \delta_{\text {ref }}\right)\left[\begin{array}{cc}
r_{\mathrm{TM}} & 0 \\
0 & r_{\mathrm{TE}}
\end{array}\right] \\
& +S_{\text {trans }} \exp \left(i \delta_{\text {trans }}\left[\begin{array}{cc}
\cos \Delta_{R 1} & \sin \Delta_{R 1} \\
-\sin \Delta_{R 1} & \cos \Delta_{R 1}
\end{array}\right]\right. \\
& \times\left[\begin{array}{cc}
t_{\mathrm{TM}}^{1} & 0 \\
0 & t_{\mathrm{TE}}^{1}
\end{array}\right]\left[\begin{array}{cc}
\cos \Delta_{01} & \sin \Delta_{01} \\
-\sin \Delta_{01} & \cos \Delta_{01}
\end{array}\right] \\
& \times\left[\begin{array}{cc}
t_{\mathrm{TM}}^{0} & 0 \\
0 & t_{\mathrm{TE}}{ }^{0}
\end{array}\right]\left[\begin{array}{cc}
\cos \Omega & \sin \Omega \\
-\sin \Omega & \cos \Omega
\end{array}\right] .
\end{aligned}
$$

The various terms in Eq. (78) have the following interpretations. Reflection is diagonal in the reflected-ray TE and TM polarization basis while diffraction is diagonal in any polarization basis. For transmission, the rightmost matrix converts the incident reflected-ray-polarization basis to the incident transmitted-ray polarization basis, and the leftmost matrix converts the final transmitted-ray polarization basis back to the final reflected-ray polarization basis. Transmission at each interface is diagonal in the transmitted-ray TE and TM polarization basis, and the middle matrix converts the 0 -interface transmitted-ray polarization basis to the 1-interface transmitted-ray polarization basis. Explicitly, the components of the scattering matrix are

$$
\begin{aligned}
S_{1}(\Theta, \Phi)= & S_{\text {diff }}+S_{\text {ref }} \exp \left(i \delta_{\text {ref }}\right) r_{\mathrm{TE}}+S_{\text {trans }} \\
& \times \exp \left(i \delta_{\text {trans }}\right)\left(t_{\mathrm{TE}}{ }^{0} t_{\mathrm{TE}} \cos \Delta_{R 1} \cos \Delta_{01} \cos \Omega\right. \\
& -t_{\mathrm{TE}}{ }^{0} t_{\mathrm{TM}}{ }^{1} \sin \Delta_{R 1} \sin \Delta_{01} \cos \Omega-t_{\mathrm{TM}}{ }^{0} t_{\mathrm{TE}}{ }^{1} \\
& \times \cos \Delta_{R 1} \sin \Delta_{01} \sin \Omega-t_{\mathrm{TM}} t_{\mathrm{TM}}{ }^{1} \sin \Delta_{R 1} \\
& \left.\times \cos \Delta_{01} \sin \Omega\right), \\
S_{2}(\Theta, \Phi)= & S_{\text {diff }}+S_{\text {ref }} \exp \left(i \delta_{\mathrm{ref}}\right) r_{\mathrm{TM}}+S_{\text {trans }} \\
& \times \exp \left(i \delta_{\text {trans }}\right)\left(t_{\mathrm{TM}}{ }^{0} t_{\mathrm{TM}}{ }^{1} \cos \Delta_{R 1} \cos \Delta_{01} \cos \Omega\right. \\
& -t_{\mathrm{TM}}{ }^{0} t_{\mathrm{TE}}{ }^{1} \sin \Delta_{R 1} \sin \Delta_{01} \cos \Omega-t_{\mathrm{TE}}{ }^{0} t_{\mathrm{TM}}{ }^{1} \\
& \times \cos \Delta_{R 1} \sin \Delta_{01} \sin \Omega-t_{\mathrm{TE}}{ }^{0} t_{\mathrm{TE}}{ }^{1} \sin \Delta_{R 1} \\
& \left.\times \cos \Delta_{01} \sin \Omega\right),
\end{aligned}
$$

$$
\begin{aligned}
S_{3}(\Theta, \Phi)= & S_{\text {trans }} \exp \left(i \delta_{\text {trans }}\right)\left(t_{\mathrm{TM}}{ }^{0} t_{\mathrm{TM}}{ }^{1} \cos \Delta_{R 1} \cos \Delta_{01}\right. \\
& \times \sin \Omega+t_{\mathrm{TE}}{ }^{0} t_{\mathrm{TM}}{ }^{1} \cos \Delta_{R 1} \sin \Delta_{01} \cos \Omega \\
& +t_{\mathrm{TE}} t_{\mathrm{TE}}{ }^{1} \sin \Delta_{R 1} \cos \Delta_{01} \cos \Omega \\
& \left.-t_{\mathrm{TM}} t_{\mathrm{TE}}{ }^{1} \sin \Delta_{R 1} \sin \Delta_{01} \sin \Omega\right),
\end{aligned}
$$

$$
\begin{aligned}
S_{4}(\Theta, \Phi)= & S_{\text {trans }} \exp \left(i \delta_{\text {trans }}\right)\left(-t_{\mathrm{TE}}{ }^{0} t_{\mathrm{TE}}{ }^{1} \cos \Delta_{R 1} \cos \Delta_{01}\right. \\
& \times \sin \Omega-t_{\mathrm{TM}}{ }^{0} t_{\mathrm{TE}}{ }^{1} \cos \Delta_{R 1} \sin \Delta_{01} \cos \Omega \\
& -t_{\mathrm{TM}}{ }^{0} t_{\mathrm{TM}}{ }^{1} \sin \Delta_{R 1} \cos \Delta_{01} \cos \Omega \\
& \left.+t_{\mathrm{TE}}{ }^{0} t_{\mathrm{TM}}{ }^{1} \sin \Delta_{R 1} \sin \Delta_{01} \sin \Omega\right) .
\end{aligned}
$$

For a spheroid that is tilted away from end-on incidence by only a small amount, the first transmitted terms of $S_{1}$ and $S_{2}$ are comparable to the diffracted and the reflected terms, and the last three transmitted terms are second-order corrections; i.e., they have two terms in the sine of a small angle. Similarly the first three terms of $S_{3}$ and $S_{4}$ are first order in the sine of a small angle, and the last term is of the third order.

Finally we obtain the ray-theory scattered intensity by multiplying Eq. (74) by its complex conjugate after inserting Eq. (78). The resulting expression is rather long. But using the shorthand notation,

$$
\begin{aligned}
D_{E} & =S_{\text {diff }} \cos \gamma, \\
D_{M} & =S_{\text {diff }} \sin \gamma, \\
R_{E} & =S_{\text {ref }} r_{\mathrm{TE}} \cos \gamma, \\
R_{M} & =S_{\text {ref }} r_{\mathrm{TM}} \sin \gamma, \\
T_{E E} & =S_{\text {trans }} t_{\mathrm{TE}}^{0} t_{\mathrm{TE}}^{1} \cos \gamma_{0}, \\
T_{E M} & =S_{\mathrm{trans}} t_{\mathrm{TE}}^{0} t_{\mathrm{TM}}^{1} \cos \gamma_{0}, \\
T_{M E} & =S_{\mathrm{trans}} t_{\mathrm{TM}}^{0} t_{\mathrm{TE}}^{1} \sin \gamma_{0}, \\
T_{M M} & =S_{\mathrm{trans}} t_{\mathrm{TM}}^{0} t_{\mathrm{TM}}^{1} \sin \gamma_{0},
\end{aligned}
$$

we obtain

$$
\begin{aligned}
& I_{\text {diff }+ \text { ref }+ \text { trans }}(\Theta, \Phi) \\
& =\frac{E_{0}^{2}}{2 \mu_{0} c} \frac{1}{k^{2} R^{2}}\left[S_{\text {diff }}^{2}+R_{E}^{2}+R_{M}^{2}\right. \\
& +\left(T_{E E}^{2}+T_{M M}^{2}\right) \cos ^{2} \Delta_{01} \\
& +2\left(R_{E} D_{E}+R_{M} D_{M}\right) \cos \delta_{\text {ref }} \\
& +2\left(T_{E E} D_{E}+T_{M M} D_{M}\right) \cos \Delta_{R 1} \cos \Delta_{01} \cos \delta_{\text {trans }} \\
& +2\left(T_{E E} R_{E}+T_{M M} R_{M}\right) \cos \Delta_{R 1} \\
& \times \cos \Delta_{01} \cos \left(\delta_{\text {trans }}-\delta_{\text {ref }}\right) \\
& +\left(T_{M E}^{2}+T_{E M}^{2}\right) \sin ^{2} \Delta_{01} \\
& +2\left(T_{M M} T_{E M}-T_{E E} T_{M E}\right) \cos \Delta_{01} \sin \Delta_{01} \\
& +2\left(T_{E E} R_{M}-T_{M M} R_{E}\right) \sin \Delta_{R 1} \\
& \times \cos \Delta_{01} \cos \left(\delta_{\text {trans }}-\delta_{\text {ref }}\right) \\
& +2\left(T_{E M} R_{M}-T_{M E} R_{E}\right) \cos \Delta_{R 1} \\
& \times \sin \Delta_{01} \cos \left(\delta_{\text {trans }}-\delta_{\text {ref }}\right) \\
& -2\left(T_{M E} R_{M}+T_{E M} R_{E}\right) \sin \Delta_{R 1} \\
& \times \sin \Delta_{01} \cos \left(\delta_{\text {trans }}-\delta_{\text {ref }}\right) \\
& +2\left(T_{E E} D_{M}-T_{M M} D_{E}\right) \sin \Delta_{R 1} \cos \Delta_{01} \cos \delta_{\text {trans }} \\
& +2\left(T_{E M} D_{M}-T_{M E} D_{E}\right) \cos \Delta_{R 1} \sin \Delta_{01} \cos \delta_{\text {trans }} \\
& \left.-2\left(T_{M E} D_{M}+T_{E M} D_{E}\right) \sin \Delta_{R 1} \sin \Delta_{01} \cos \delta_{\text {trans }}\right] \text {. }
\end{aligned}
$$

The various terms in Eq. (82) have the following physical interpretations. The second lines are the 
individual diffracted, reflected, and transmitted intensities for scattering by a sphere or an end-on spheroid. The reflected and transmitted intensities in the $\Theta, \Phi$ direction depend on the polarization state $(E$ or $M)$ of the incident beam. The fourth and seventh lines are the diffraction-reflection interference, the diffraction-transmission interference, and the reflection-transmission interference for scattering by a sphere or end-on spheroid. The eighth and ninth lines are the cross-polarization contribution to the transmitted intensity, which is due solely to the rotation of the $\widehat{\mathrm{TE}}_{t 0}$ and $\widehat{\mathrm{TE}}_{i 1}$ polarization directions. For scattering in the near-forward direction by a spheroid tilted a small amount from end-on incidence these two terms are second-order corrections because $\sin \Delta_{01}$ is small and because the TE and TM Fresnel coefficients are nearly equal for small angles of incidence. ${ }^{44}$ The tenth through fifteenth lines are the cross-polarization contribution to the reflectiontransmission interference. The sixteenth through eighteenth lines are the cross-polarization contribution to the diffraction-transmission interference. For scattering in the near-forward direction by a spheroid tilted a small amount from end-on incidence these are also second-order corrections, either because of sine-squared factors or because of a single sine factor and the near equality of the TE and TM Fresnel coefficients. Because diffraction and reflection are both diagonal in the reflected-ray basis [see Eq. (78)] there is no cross-polarized diffractionreflection interference. The cross-polarized contributions to Eq. (84) become comparable to the second through fourth lines for a highly eccentric spheroid tilted substantially away from end-on incidence and for rays with large angles of incidence at the points of entrance to and exit from the spheroid.

\section{Computation of the Scattered Intensity}

It has already been shown in part I that the diffracted plus reflected electric field is directly expressible in terms of $\Theta$ and $\Phi$. But because of the complexity of both the spheroid shape and the refraction geometry, the transmitted electric field is not directly expressible in terms of $\Theta$ and $\Phi$. This suggests the following strategy for computing the ray-theory intensity of Eq. (84). We first generate a dense grid of $r_{0}{ }^{\prime}$ and $\xi_{0}{ }^{\prime}$ values, i.e., $\Delta r_{0}{ }^{\prime}=0.005$ and $\Delta \xi_{0}{ }^{\prime}=1^{\circ}$. Then for each $r_{0}{ }^{\prime}, \xi_{0}{ }^{\prime}$, we compute the scattering angles $\Theta$ and $\Phi$. We then numerically perform the derivatives in Eq. (46) and obtain the magnitude, phase, and polarization direction of the transmitted ray. For each $r_{0}{ }^{\prime}, \xi_{0}{ }^{\prime}$ we also check whether the ray is totally internally reflected by the use of Eq. (33). If it is not totally internally reflected, we test whether it exits on the shadowed or the lit side of the spheroid with Eqs. (25) and (26). Knowing $\Theta$ and $\Phi$ for each transmitted ray, we then calculate the reflected and diffracted electric fields for those scattering angles, and finally we compute the scattered intensity of Eq. (84).

In order to test our numerical procedure, first $b=$ $a$ was set in the computer program, and the results were compared against ray scattering by a sphere as given by the analytical formulas in Refs. 3 and 4 .

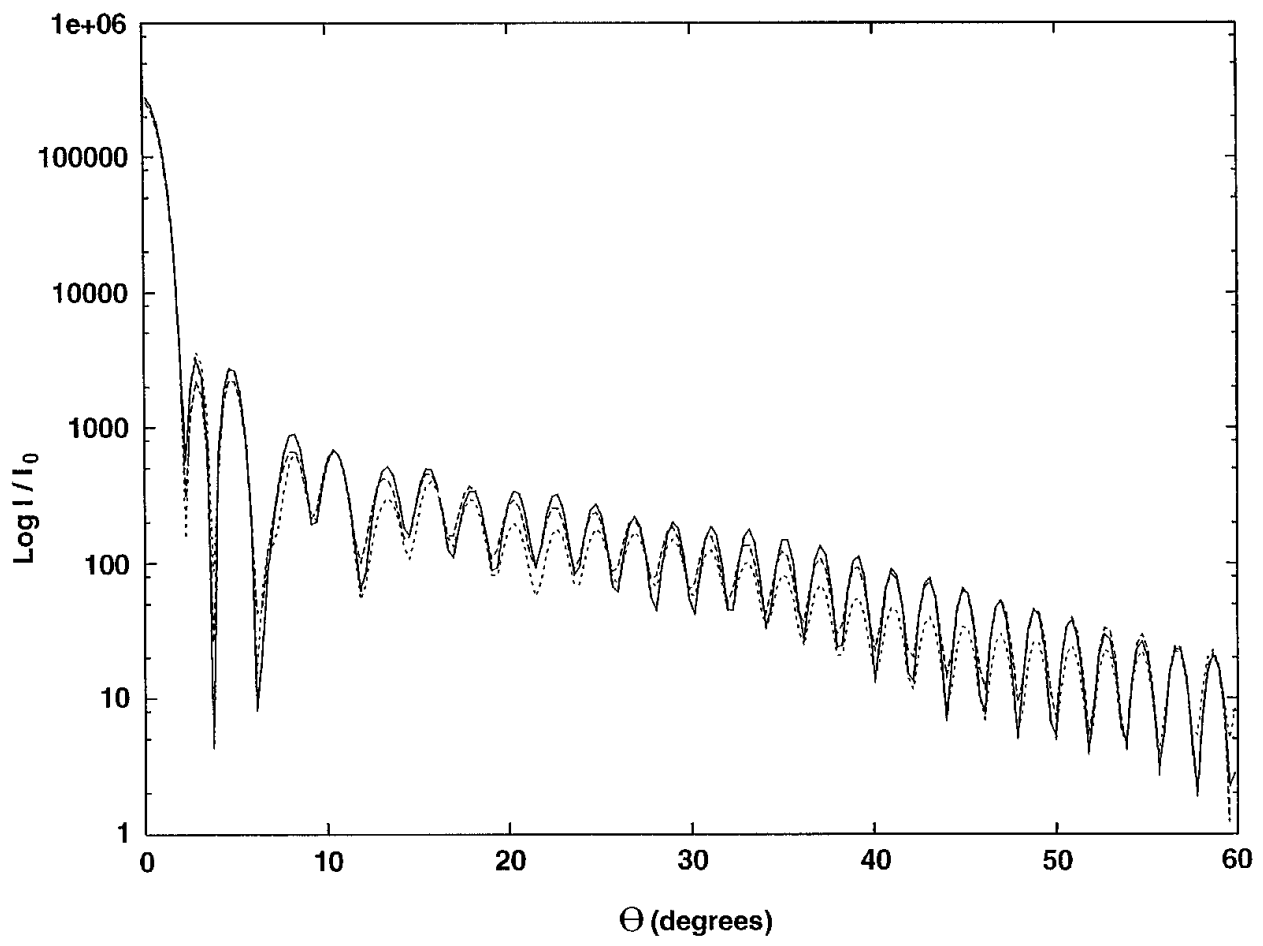

Fig. 8. Intensity as a function of the scattering angle $\Theta$ with $\Phi=0^{\circ}$ for a plane wave with $\lambda=0.6328 \mu \mathrm{m}$ and $\chi=90^{\circ}$ incident upon a sphere with $a=10.071 \mu \mathrm{m}$ and $n=1.333$. The solid curve is the Lorenz-Mie theory result, the dashed curve is Eq. (84), and the dotted curve is the generalized eikonal approximation of Ref. 45. 
The results matched exactly. For scattering by a sphere or an end-on spheroid, Eq. (46) reduces to

$$
\Delta=\frac{\partial \Theta}{\partial r_{0}{ }^{\prime}} \text {. }
$$

The agreement between our computer program and the numerical implementation of the analytical formulas for sphere scattering in Refs. 3 and 4 verifies that we have chosen our grid of $r_{0}{ }^{\prime}$ values to be fine enough to perform the numerical derivative in Eq. (85)

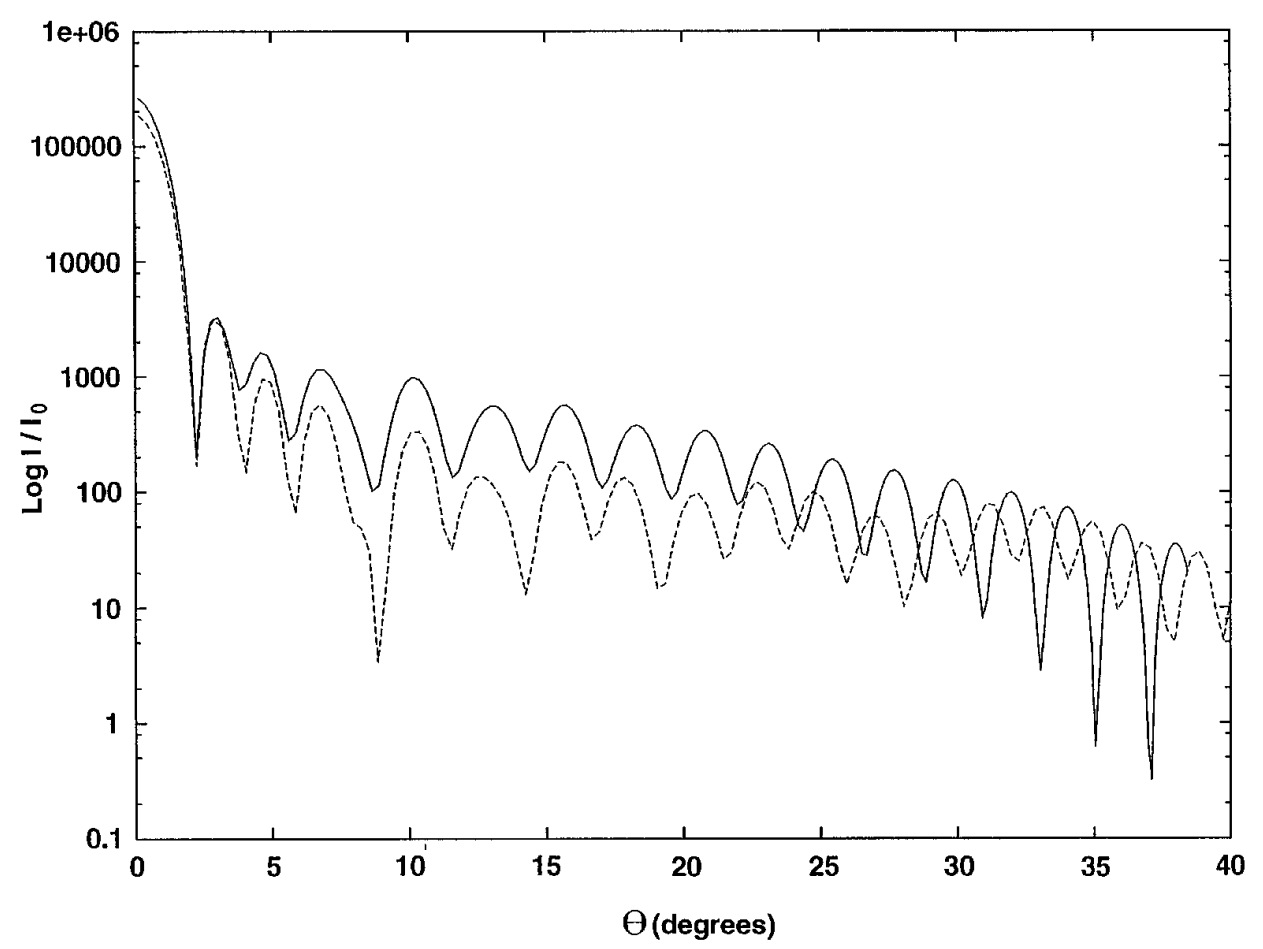

(a)

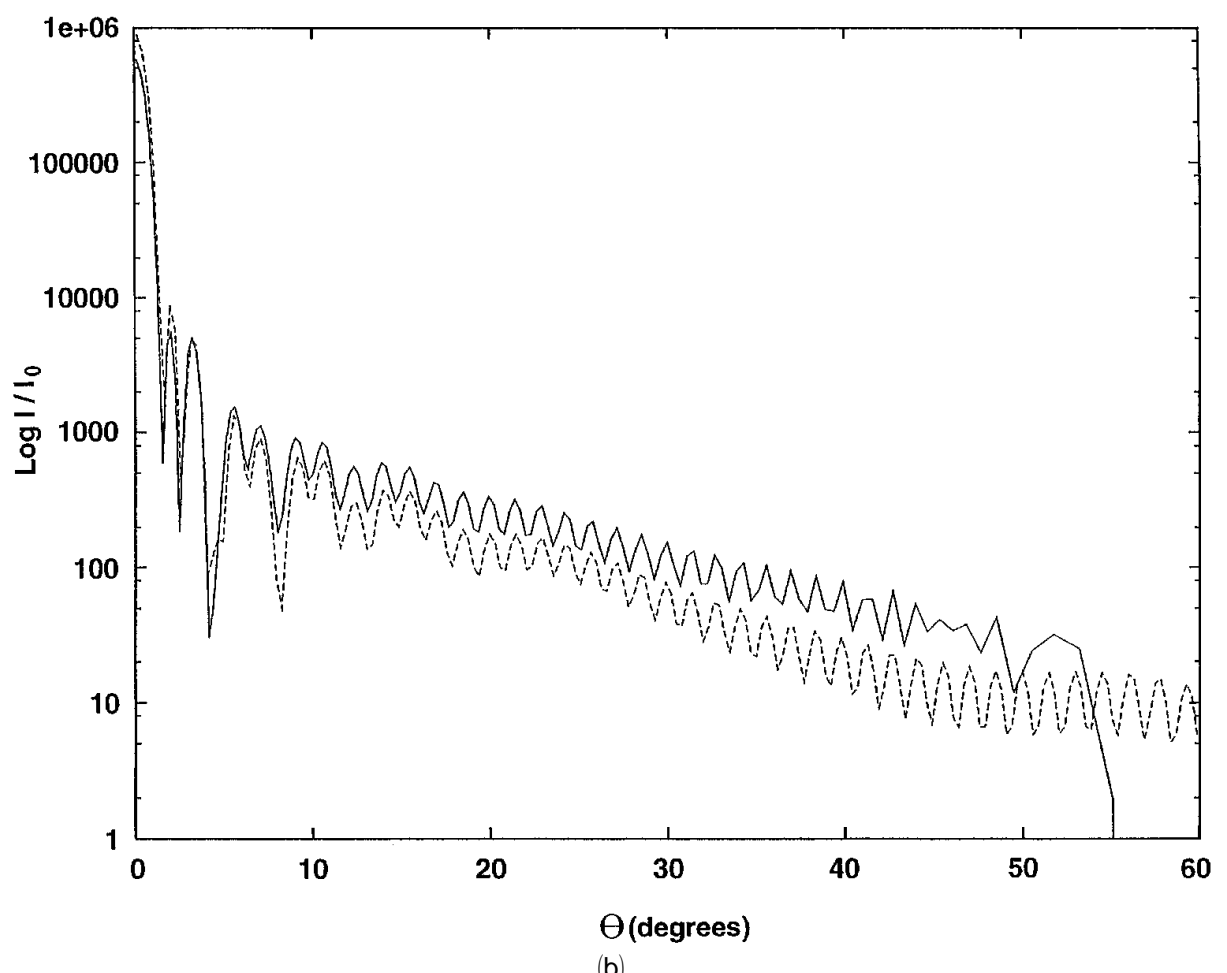

(b)

Fig. 9. Intensity as a function of the scattering angle $\Theta$ with $\Phi=0^{\circ}$ for a plane wave with $\lambda=0.6328 \mu \mathrm{m}$ and $\chi=90^{\circ}$ incident upon a spheroid with $a=10.071 \mu \mathrm{m}, n=1.333$, and $b / a=1.5 \operatorname{In}(\mathrm{a})$ the spheroid is in the end-on orientation, with $\theta=0^{\circ}$ and $\phi=0^{\circ}$. In $(\mathrm{b})$ it is in the side-on orientation, with $\theta=90^{\circ}$ and $\phi=0^{\circ}$. The solid curve is Eq. (84), and the dashed curve is the generalized eikonal approximation of Ref. 47. 
accurately. Our computed result for $b=a=10.07$ $\mu \mathrm{m}$ also closely approximates Lorenz-Mie theory, as is shown in Fig. 8. Also shown in Fig. 8 is the generalized eikonal approximation. ${ }^{45}$ The eikonal approximation has been shown to be an accurate approximation in the short-wavelength limit. It has the feature that it slightly underestimates the peak intensity of the reflection-transmission interference structure for $20^{\circ} \leq \Theta \leq 50^{\circ}$ for $n=1.33$.

We next considered the end-on spheroid case $\lambda=$ $0.6328 \mu \mathrm{m}, n=1.33, b / a=1.5, a=10.0 \mu \mathrm{m}, \chi=90^{\circ}$, and $\theta=0^{\circ}$ and compared our results with those in figure 8 of Ref. 21, in which the ray-theory intensity was calculated with another method. Again the results matched exactly. ${ }^{46}$ We also compared our results with the generalized eikonal approximation for $a=10.07 \mu \mathrm{m} .{ }^{47}$ The comparison is shown in Fig. 9(a). For $\Theta \lesssim 30^{\circ}$ ray theory and the generalized eikonal approximation give similar results, with the generalized eikonal approximation again slightly underestimating the reflection-transmission interference for $\theta \geqslant 10^{\circ}$. The critical angle for total internal reflection occurs at a scattering angle of approximately $\theta \approx 38^{\circ}$. Thus ray theory is expected to be inaccurate for $\Theta \geqslant 33^{\circ}$ because of our neglect of the Fock transition ${ }^{48}$ in the transmitted electric field. This neglect may be the cause of ray theory becoming out of phase with the eikonal approximation for $\Theta \geqslant 25^{\circ}$.

In Fig. 9(b), ray theory is compared with the generalized eikonal approximation for side-on incidence with $\lambda=0.6328 \mu \mathrm{m}, n=1.333, b / a=1.5, a=$ $10.071 \mu \mathrm{m}, \theta=90^{\circ}, \phi=0^{\circ}$, and $\chi=90^{\circ}$ for scattering in the $\Phi=0^{\circ}$ plane. The cross section of the spheroid in the $x z$ plane (i.e., $\Phi=0^{\circ}$ ) resembles an oblate spheroid with $b / a=0.667$ in the end-on incidence configuration. For this configuration and $\Phi=0^{\circ}$, each transmitted ray participates in two focusing caustics. Again ray theory and the generalized eikonal approximation give similar results, with the generalized eikonal approximation slightly underestimating the reflection-transmission interference for $15^{\circ} \lesssim \Theta \lesssim 40^{\circ}$. The critical angle for total internal reflection occurs at a scattering angle of approximately $\Theta \approx 55^{\circ}$. Thus our neglect of the Fock transition causes ray theory to be inaccurate for $\Theta \geq 50^{\circ}$.

In Fig. 10 ray theory is compared with the exact solution of the plane-wave-spheroid problem with the method of Ref. 49. The comparison was made for end-on [Fig. 10(a)] and side-on [Fig. 10(b)] incidence for $\lambda=0.6328 \mu \mathrm{m}, n=1.333, b / a=1.5, a=3.021$ $\mu \mathrm{m}$, and $\chi=90^{\circ}$ for scattering in the $\Phi=0^{\circ}$ plane. This corresponds to a spheroid-size parameter of $2 \pi / \lambda=30.0$, which is at the lower end of the region of applicability of ray theory. In Fig. 10(a) the comparison is good for $\Theta \lesssim 30^{\circ}$, and in Fig. 10(b) the comparison is good for $\Theta \leqslant 50^{\circ}$. We consider the general agreement between our results and the generalized eikonal model for $2 \pi a / \lambda \approx 100$ and the exact solution for $2 \pi a / \lambda \approx 30$ as an additional check of the correctness of our method.

It is of great interest to compute the crosspolarization contributions to the scattered intensity of Eq. (84). Unfortunately, this cannot be done reliably until a complete solution to the problem of the number of caustic participations of an arbitrary transmitted ray is obtained. This problem is currently under study. It is also of great interest to compare our results with experimental data. Although much experimental spheroid scattering data concerning the generalized rainbow in the backward hemisphere exists, ${ }^{16,50-53}$ experimental spheroid scattering data in the near-forward direction appear to be sparse. ${ }^{54}$

Last, it has been pointed out by a number of authors ${ }^{8,11,12}$ that both the T-matrix solution and the spheroidal wave-function solution of the problem of scattering a plane wave by an arbitrarily oriented spheroid possess numerical difficulties for prolate spheroids with large $b / a$ ratios. In ray theory, we have found that a number of alternatives occur for

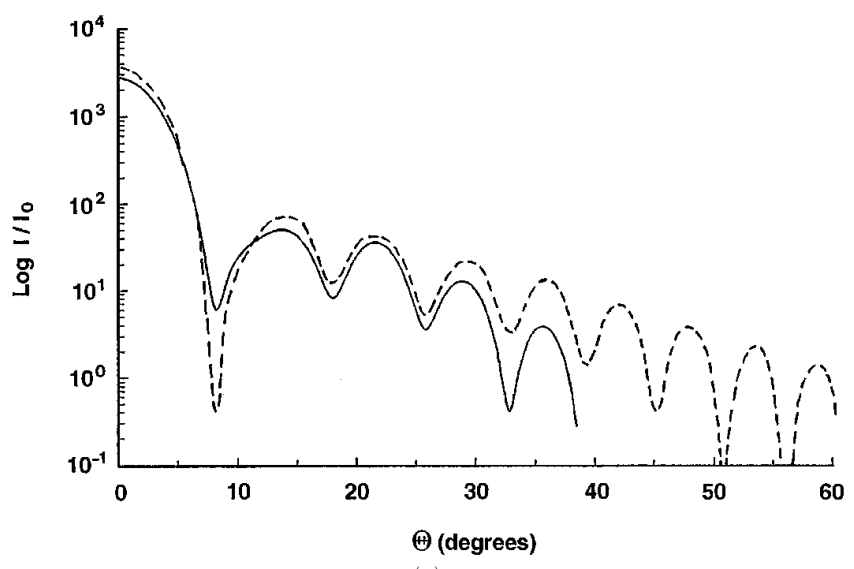

(a)

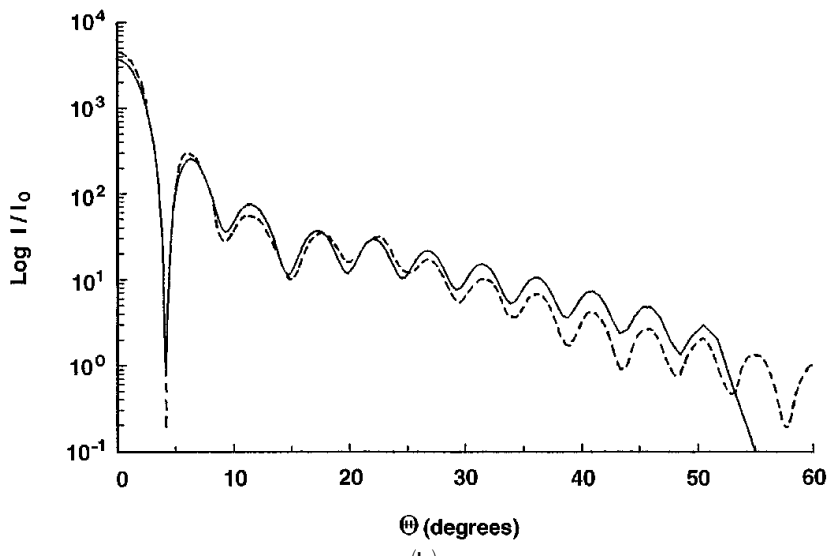

(b)

Fig. 10. Intensity as a function of the scattering angle $\Theta$ with $\Phi$ $=0^{\circ}$ for a plane wave with $\lambda=0.6328 \mu \mathrm{m}$ and $\chi=90^{\circ}$ incident upon a spheroid with $a=3.021 \mu \mathrm{m}, n=1.333$, and $b / a=1.5$. In (a) the spheroid is in the end-on orientation, with $\theta=0^{\circ}$ and $\phi=$ $0^{\circ}$. In (b) it is in the side-on orientation, with $\theta=90^{\circ}$ and $\phi=$ $0^{\circ}$. The solid curve is Eq. (84), and the dashed curve is the exact solution of Ref. 49 . 
rays transmitted through large $b / a$ prolate spheroids. These include the point of exit of the ray being on either the shadowed or the lit side of the spheroid, a complicated caustic structure that dictates the phase of the transmitted electric field, and the existence of more than one Fock transition of the transmitted electric field as a function of scattering angle [see Fig. $3(\mathrm{~h})$, for example]. It would be of interest to determine whether any of these complexities for eccentric prolate spheroids are related to the difficulties in computation for the associated wave-scattering problem.

The work was supported in part by the National Aeronautics and Space Administration grant NCC 3-204. I wish to thank T. W. Chen and L. Yang of New Mexico State University for permitting me to quote some of their results before publication. I also thank J. P. Barton of the University of Nebraska, Lincoln, for providing me with the exact scattering data quoted in Fig. 10.

\section{References and Notes}

1. W. D. Bachalo, "Method for measuring the size and velocity of spheres by dual-beam light-scatter interferometry," Appl. Opt. 19, 363-370 (1980).

2. W. D. Bachalo and M. J. Houser, "Phase/Doppler spray analyzer for simultaneous measurements of drop size and velocity distributions," Opt. Eng. 23, 583-590 (1984).

3. W. J. Glantschnig and S.-H. Chen, "Light scattering from water droplets in the geometrical optics approximation," Appl. Opt. 20, 2499-2509 (1981).

4. A. Ungut, G. Gréhan, and G. Gouesbet, "Comparisons between geometrical optics and Lorenz-Mie theory," Appl. Opt. 20, 2911-2918 (1981).

5. S. V. Sanker and W. D. Bachalo, "Response characteristics of the phase Doppler particle analyzer for sizing spherical particles larger than the light wavelength," Appl. Opt. 30, 1487-1496 (1991).

6. M. Schneider and E. D. Hirleman, "Influence of internal refractive index gradients on size measurements of spherically symmetric particles by phase Doppler anemometry," Appl. Opt. 33, 2379-2388 (1994).

7. S. Asano and G. Yamamoto, "Light scattering by a spheroidal particle," Appl. Opt. 14, 29-49 (1975); 15, 2028(E) (1976).

8. S. Asano, "Light scattering properties of spheroidal particles," Appl. Opt. 18, 712-723 (1979).

9. P. Barber and C. Yeh, "Scattering of electromagnetic waves by arbitrarily shaped dielectric bodies," Appl. Opt. 14, 2864$2872(1975)$.

10. P. W. Barber and S. C. Hill, Light Scattering by Particles: Computational Methods (World Scientific, Singapore, 1990), Chap. 3.

11. J. P. Barton and D. R. Alexander, "Electromagnetic fields for an irregularly shaped near-spherical particle illuminated by a focused laser beam," J. Appl. Phys. 69, 7973-7986 (1991).

12. G. R. Fournier and B. T. N. Evans, "Approximation to extinction efficiency for randomly oriented spheroids," Appl. Opt. 30, 2042-2048 (1991).

13. K. Sassen, "Angular scattering and rainbow formation in pendant drops," J. Opt. Soc. Am. 69, 1083-1089 (1979).

14. A. B. Fraser, "Why can the supernumerary bows be seen in a rain shower?" J. Opt. Soc. Am. 73, 1626-1628 (1983).

15. G. P. Können, "Appearance of supernumeraries of the second rainbow in rain showers," J. Opt. Soc. Am. A 4, 810-816 (1987).

16. P. L. Marston and E. H. Trinh, "Hyperbolic umbilic diffraction catastrophe and rainbow scattering from spheroidal drops," Nature 316, 529-531 (1984).

17. J. F. Nye, "Rainbow scattering from spheroidal drops-an explanation of the hyperbolic umbilic foci," Nature 316, 531-532 (1984).

18. P. L. Marston, "Cusp diffraction catastrophe from spheroids: generalized rainbows and inverse scattering," Opt. Lett. 10, 588-590 (1985).

19. J. F. Nye, "Rainbows from ellipsoidal water drops," Proc. R. Soc. London Ser. A 438, 397-417 (1992).

20. M. Bottlinger and H. Umhauer, "Modeling of light scattering by irregularly shaped particles using a ray-tracing method," Appl. Opt. 30, 4732-4738 (1991).

21. E. A. Hovenac, "Calculation of far-field scattering from nonspherical particles using a geometrical optics approach,"Appl. Opt. 30, 4739-4746 (1991).

22. J.A. Lock, "Ray scattering by an arbitrarily oriented spheroid. I. Diffraction and specular reflection," Appl. Opt. 35, 500$514(1996)$.

23. H. C. van de Hulst, Light Scattering by Small Particles (Dover, New York, 1981), pp. 34-35, 46, 57.

24. M. Kerker, The Scattering of Light and Other Electromagnetic Radiation (Academic, New York, 1969), p. 498.

25. C. F. Bohren and D. R. Huffman, Absorption and Scattering of Light by Small Particles (Wiley, New York, 1983), pp. 383, 402-403.

26. Ref. 24, Chap. 10.

27. Ref. 25, Sect. 5.3-5.7 and 8.2.

28. Ref. 25, Sect. 8.3.

29. Ref. 25, Sect. 8.4.

30. S. Asano and M. Sato, "Light scattering by randomly oriented spheroidal particles," Appl. Opt. 19, 962-974 (1980).

31. J. B. Keller and H. B. Keller, "Determination of reflected and transmitted fields by geometrical optics," J. Opt. Soc. Am. 40, 48-52 (1950).

32. D. G. Burkhard and D. L. Shealy, "Simplified formula for the illuminance in an optical system," Appl. Opt. 20, 897-909 (1981).

33. A. M. Kassim, D. L. Shealy, and D. G. Burkhard, "Caustic merit function for optical design," Appl. Opt. 28, 601-606 (1989).

34. J. A. Lock and E. A. Hovenac, "Internal caustic structure of illuminated liquid droplets,” J. Opt. Soc. Am. A 8, 1541-1552 (1991).

35. Ref. 23, Secs. 12.22 and 12.33 .

36. E. Hecht, Optics, 2nd ed. (Addison-Wesley, Reading, Mass., 1987), Sect. 5.2.1.

37. R. W. Boyd, "Intuitive explanation of the phase anomaly of focused light beams," J. Opt. Soc. Am. 70, 877-880 (1980).

38. J. A. Lock and T. A. McCollum, "Further throughts on Newton's zero-order rainbow,” Am. J. Phys. 62, 1082-1089 (1994).

39. M. V. Berry and C. Upstill, "Catastrophe optics: morphologies of caustics and their diffraction patterns," Prog. Opt. 18, 257-346 (1980), figure 2.5.

40. M. V. Berry, "Waves and Thom's theorem," Adv. Phys. 25, 1-26 (1976).

41. D. L. Shealy and D. G. Burkhard, "Caustic surfaces and irradiance for reflection and refraction from an ellipsoid, elliptic paraboloid, and elliptic cone," Appl. Opt. 12, 29552959 (1973).

42. Ref. 23 , Sec. 4.41 .

43. Ref. 25 , Sec. 3.2 .

44. Ref. 38, figure 4.20-4.22.

45. T. W. Chen, "Simple formula for light scattering by a large spherical dielectric," Appl. Opt. 32, 7568-7571 (1993).

46. Figure 8 of Ref. 21 also contains the contributions of 2 , 4, and 6 internal reflections. When these are removed, our result 
agrees exactly [E. A. Hovenac, NASA Lewis Research Center, Cleveland, Oh. 44115 (personal communication, August 1994)].

47. T. W. Chen and L. Yang, New Mexico State University, Las Cruces, N.M. 88003 (personal communication, December 1994).

48. H. M. Nussenzveig, "High-frequency scattering by a transparent sphere. I. Direct reflection and transmission," J. Math. Phys. 10, 82-124 (1969).

49. J. P. Barton, "Internal and near-surface electromagnetic fields for a spheroidal particle with arbitrary illumination," Appl. Opt. 34, 5542-5551 (1995).

50. H. J. Simpson and P. L. Marston, "Scattering of white light from levitated oblate water drops near rainbows and other diffraction catastrophes," Appl. Opt. 30, 3468-3473, 3547 (1991).

51. G. Kaduchak, P. L. Marston, and H. J. Simpson, " $E_{6}$ diffrac- tion catastrophe of the primary rainbow of oblate water drops: observations with white-light and laser illumination," Appl. Opt. 33, 4691-4696, 4961 (1994).

52. G. Kaduchak and P. L. Marston, "Hyperbolic umbilic and $\mathrm{E}_{6}$ diffraction catastrophes associated with the secondary rainbow of oblate water drops: observations with laser illumination,"Appl. Opt. 33, 4697-4701 (1994).

53. P. L. Marston and G. Kaduchak, "Generalized rainbows and unfolded glories of oblate drops: organization for multiple internal reflections and extension of cusps into Alexander's dark band," Appl. Opt. 33, 4702-4713 (1994).

54. The only published photograph to my knowledge is in A. Ashkin and J. M. Dziedzic, "Observation of light scattering from nonspherical particles using optical levitation," Appl. Opt. 19, 660-668 (1980), figure 18b. 